\title{
Cratering Rates on the Galilean Satellites
}

\author{
Kevin Zahnle and Luke Dones \\ NASA Ames Research Center, MS 245-3, Moffett Field, California 94035 \\ E-mail: kzahnle@mail.arc.nasa.gov \\ and \\ Harold F. Levison \\ Southwest Research Institute, 1050 Walnut St., Boulder, Colorado 80302
}

Received March 16, 1998; revised July 20, 1998

We exploit recent theoretical advances toward the origin and orbital evolution of comets and asteroids to obtain revised estimates for cratering rates in the jovian system. We find that most, probably more than $\mathbf{9 0 \%}$, of the craters on the Galilean satellites are caused by the impact of Jupiter-family comets (JFCs). These are comets with short periods, in generally lowinclination orbits, whose dynamics are dominated by Jupiter. Nearly isotropic comets (long period and Halley-type) contribute at the 1-10\% level. Trojan asteroids might also be important at the $1-10 \%$ level; if they are important, they would be especially important for smaller craters. Main belt asteroids are currently unimportant, as each 20 - $\mathrm{km}$ crater made on Ganymede implies the disruption of a $200-\mathrm{km}$ diameter parental asteroid, a destruction rate far beyond the resources of today's asteroid belt.

Twenty-kilometer diameter craters are made by kilometersize impactors; such events occur on a Galilean satellite about once in a million years. The paucity of $20-\mathrm{km}$ craters on Europa indicates that its surface is of order $10 \mathrm{Ma}$. Lightly cratered surfaces on Ganymede are nominally of order $0.5-1.0 \mathrm{Ga}$. The uncertainty in these estimates is about a factor of five. Callisto is old, probably more than $4 \mathrm{Ga}$. It is too heavily cratered to be accounted for by the current flux of JFCs. The lack of pronounced apex-antapex asymmetries on Ganymede may be compatible with crater equilibrium, but it is more easily understood as evidence for nonsynchronous rotation of an icy carapace. $\odot 1998$ Academic Press

Key Words: comets; impacts; craters; Ganymede; Europa; Jupiter.

\section{INTRODUCTION}

The four Galilean satellites present one of the few strong arguments for a planned solar system. They orbit Jupiter like an evolutionary sequence, with the innermost, Io, being in one sense the youngest solid body in the solar sys- tem-it has no known impact craters-but in another sense one of the most thoroughly evolved. At the other extreme sits cold-hearted Callisto, in some sense a deeply primitive world where the inner fires never quite got lit. Any external sign of the solid state convection that keeps it cold has been erased.

In between we find Europa and Ganymede, interpolated worlds that will command the bulk of our attention. Europa is an ice-covered but mostly rocky world with very few unambiguous impact craters. Speculations abound (Hoagland 1980, Clarke 1982, Reynolds et al. 1983, Carr et al. 1998, Pappalardo et al. 1998, Geissler et al. 1998, Sullivan et al. 1998), but what seems clear enough is that whatever may lurk beneath the ice, the surface itself is sparsely cratered and looks young. Assigning an age to the surface is one of the tasks before us here.

Ganymede's surface is older than Europa's, but overall Ganymede may have more in common with Europa than it has with its lost twin Callisto. Like Europa it is thoroughly differentiated, such that it is now a layered body of ice, rock, and iron (Anderson et al. 1997). Ganymede was certainly active in the past. When was this? The assumption has been that Ganymede is as old as the Moon. In this view, the relatively young Gilgamesh basin is to Ganymede roughly as the Orientale basin is to the Moon. It is therefore often assumed that Gilgamesh has the same $\sim 3.8 \mathrm{Ga}$ age as Orientale (Shoemaker et al. 1982, Neukum et al. 1997). The underlying presumption is that the cratering histories of the inner and outer solar systems were the same. What is odd about this assumption is that the objects that are responsible for modern craters on Ganymede are the Jupiter-family comets (JFCs), a class of object that currently has negligible impact in the inner solar system (Shoemaker and Wolfe 1982, Levison and Duncan 1997).

Ganymede provides us with a second chronometer that 
is independent of the cratering rate. Ganymede has a live magnetic field that presumably has its roots in a convecting iron core. As it is difficult to maintain a hot convecting iron core in a moon like Ganymede for more than $1 \mathrm{Ga}$, it is possible that the present episode of cooling began in the cosmically recent past (Stevenson 1996). Does the surface record this event? Stevenson (1996) suggested that it does. This will prove to be the most difficult question for us to address here, because the uncertainties in the cratering rate span the age of the solar system, but the nominal result is intriguing.

This paper will be much more narrowly focused than it could be. It is in essence a revision of Shoemaker and Wolfe's (1982; hereafter SW82) study of cratering rates in the jovian system. We will rely heavily on three recent papers in numerical dynamics: Levison and Duncan (1997; hereafter LD97), who model the evolution of Kuiper Belt objects into Jupiter-family comets; Levison et al. (1997; LSS97), who model the trojans; and Gladman et al. (1997), who model the ejection of asteroids from resonances in the asteroid belt. We also exploit the LD97 dataset to make new analyses of Jupiter encounters.

Most of the impacts in the jovian system are by comets of the Jupiter family (SW82). Comet Shoemaker-Levy 9 provides a recent and relevant example. JFCs are now generally thought to originate in the Kuiper Belt (Duncan et al. 1988; LD97). They are distinctive for their generally low, prograde inclinations, which is the trait they share with the Kuiper Belt objects. The JFCs appear to be genetically distinct from the long period comets (LPCs, i.e., Oort cloud comets) and the Halley-type comets (HTCs). The latter refers to comets that, like $\mathrm{P} / \mathrm{Halley}$, have relatively short periods but a wide distribution of inclinations, including many that are retrograde. HTCs were once lumped together with the JFCs as short period comets, but now appear to be better regarded as the short period tail of the distribution of Oort cloud comets. In this study we will consider all three classes of comets, but we will emphasize the JFCs, as these are much the most important for cratering in the jovian system.

We will also consider asteroids. The more important source is the trojan asteroids, objects co-orbiting with Jupiter in the L4 and L5 stable resonances (roughly $\pm 60^{\circ}$ of Jupiter). Escaped trojans evolve in orbits essentially similar to JFCs (Rabe 1972). The other source of asteroids, the main belt, is harder to assess. However, it is a simple matter to show that the main belt is not currently an important source of cratering in the jovian system, whatever the case may have been 4 billion years ago.

The plan of the paper is as follows: In Section 2 we will discuss Jupiter-family comets. We will use LD97 and some additional analyses of the data generated by LD97 to characterize the orbits of JFCs encountering Jupiter. Here we construct a Monte Carlo model to simulate the interaction of comets from these orbital distributions with Jupiter and its satellites. From this model we deduce impact velocities and relative impact probabilities normalized to the impact rate on Jupiter. We discuss the craters produced by these impacts. We next discuss how the size and numbers of comets encountering Jupiter are calibrated to the size and numbers of JFCs we observe in the inner solar system. We do this three ways. It is also possible to independently calibrate the flux of JFCs at Jupiter by considering the three JFCs known to have made close historical encounters with Jupiter-this sets a very interesting lower limit on the current impact rate. We close the section on JFCs with a discussion of the uncertainties in our derived cratering rates. In Section 3 we do the same things for the nearly isotropic comets (long period and Halley-type). Section 4 addresses the asteroids, especially the trojans. Section 5 compares our results to those from previous studies. In Section 6 we address apex-antapex cratering asymmetries produced by the tendency of impacts to occur more frequently and more energetically on the leading face of a synchronously rotating satellite. Section 7 provides brief discussions of surface ages of the Galilean satellites. The paper closes with a few reiterated remarks.

\section{JUPITER-FAMILY COMETS}

The Jupiter-family refers to short period (typically less than 20 years) comets in low inclination orbits controlled by interactions with Jupiter. A more precise definition can be stated in terms of the Tisserand parameter with respect to Jupiter (Carusi et al. 1987, Shoemaker et al. 1994, Levison and Duncan 1994, Levison 1996),

$$
T=1 / A+2 \sqrt{A\left(1-e^{2}\right)} \cos i,
$$

where $A$ is the comet's semimajor axis in units of Jupiter's semimajor axis, $e$ is the eccentricity, and $i$ the inclination with respect to Jupiter's orbital plane. The Tisserand parameter, which is approximately conserved on interactions with Jupiter, is a measure of Jupiter's control over the orbit. Shoemaker et al. (1994) and Levison (1996) suggested that JFCs are best defined by $2<T \leq 3$; the great majority of JFCs have $2.8<T \leq 3$. Ecliptic comets with $T>3$ do not cross Jupiter's orbit, and comets with $T<2$ are "nearly isotropic" (Levison 1996). It is rare for a JFC to evolve into an orbit with $T<2$ (Levison and Duncan 1994, 1997). There are 145 or so known JFCs and a great many more that are yet to be discovered, but currently there are only five or six active known Earth-crossers.

It was once thought that JFCs were simply captured from the Oort cloud by unlikely gravitational encounters with Jupiter. This viewpoint was integral to SW82's analysis of cratering by LPCs. This hypothesis became testable with modern computers and modern dynamical codes, and it 
appears to have failed: capture is incapable of producing a relatively flattened prograde distribution of orbits. Instead, a parental low inclination source is required. Thus Duncan et al. (1988) were led to re-invent the Kuiper Belt as the source region for JFCs. Subsequent work, both theoretical and observational, has served to confirm this hypothesis. There is an observable Kuiper Belt, populated by at least three score known objects as we write (Dones 1997), and the orbital characteristics of its escapees provide a good match to the observed population of JFCs (LD97).

LD97 follow the evolution of $>2000$ test particles for 1 $\mathrm{Ga}$ from their origins in the Kuiper Belt to their eventual destinies. As a class, LD97 refer to these objects as "ecliptic comets," of which the JFCs are a subset. Other subsets include "Centaurs," of which Chiron is an example, and $\mathrm{P} /$ Encke, a short period comet with $T>3$ that has set its controls for the heart of the Sun. Most test particles are ultimately either injected into the Oort cloud or (more probably) ejected into the pathless wilderness of interstellar space. A few hit planets $(2 \%)$ and a few hit the Sun $(1 \%)$. But during their travels about a third of the test particles spend some time (average 7\%) as JFCs with perihelia $q<2.5 \mathrm{AU}$. The simulation is not perfect-it does not explicitly include Earth and Venus, and it is (therefore?) unable to produce $\mathrm{P} /$ Encke (its orbit is deep enough that Encke revolves beyond Jupiter's reach), and it does not necessarily sample the orbits in the Kuiper Belt in the same proportions that the solar system does-but it is good enough to inspire a fair degree of confidence.

As noted above, although the main purposes of LD97 were to demonstrate that the Kuiper Belt is the source of the JFCs and to test whether the Kuiper Belt could be the source of the HTCs, their study also provides the best available and least biased description of the population of ecliptic comets in the vicinity of Jupiter. In particular, LD97 calculate directly the number of impacts on Jupiter (by counting the number of hits), and by exploiting their inventory of orbits they are able to estimate the number of impacts on Earth using Öpik's formulae. LD97 calibrate these and other impact rates to the number of active JFCs observed near the Earth. However, they do not compute impact rates on the satellites and they do not assign masses to their comets, a problem that is central to our concerns here. Calibrating the mass distribution is the key step to applying LD97's simulation to impact rates in the jovian system; we will revisit this issue.

Two other recent papers address impact rates on Jupiter (Nakamura and Yoshikawa 1995, Kary and Dones 1996); the former also address impact rates on the moons. Nakamura and Yoshikawa (hereafter NY95) numerically integrate the orbits of the 165 known short period comets then known (they do not distinguish between Halley-type and Jupiter-family) for 4400 years. Because comets with small perihelia are much more likely to be seen, and 4400 years is a very short time, the simulation is obviously biased against comets with distant perihelia. To correct for this NY95 invoke "invisibility factors" assigned to different categories of orbit (sorted by Tisserand parameter) in order to reconstruct a more likely steady-state population. In particular, more or less circular orbits with $T \approx 3$ are severely undercounted. For these orbits NY95 estimate an "invisibility" of 18 , by which they mean that 1 in 18 are in orbits with favorable $(q<2 \mathrm{AU})$ perihelia. To estimate the impact rate on Jupiter, NY95 use an "invisibility" of 7.4 as an average correction factor for all short period comets (i.e., they multiply the result they obtain for the $165 \mathrm{SP}$ comets by 7.4). Overall, this approach is similar to SW82's, who also reconstruct the population in invisible orbits; their global correction factor was 3.9.

Kary and Dones (hereafter KD96), in a study designed to estimate the frequency of events like Shoemaker-Levy 9, were looking for the fraction of comets striking Jupiter that were orbiting Jupiter at the time. They assumed an initial distribution of 49,000 JFC orbits and integrated these for $\sim 10^{5}$ years. They found that the inclinations quickly $\left(<10^{4}\right.$ years) settled into a steady state distribution, but eccentricities continually evolved. This failure to relax to a steady state is to be expected of a model with sinks but no source. Because the initial distribution is arbitrary, it is not clear that the computed eccentricity evolution is universal or unique to the assumed initial conditions.

Given a population of JFCs, we will (i) simulate JFC orbits as they are perceived by the Galilean satellites, and use these simulated orbits to estimate the impact velocities and Öpik impact probabilities relative to impacts on Jupiter; (ii) estimate the mass of a comet required to excavate 10- and 20-km diameter craters; and (iii) calibrate the LD97 impactor flux to the mass and number of JFCs in the inner solar system. The result is the current steady-state cratering rates on the Galilean satellites.

\subsection{Simulated Orbits}

From the perspective of a Galilean satellite, the Jupiterfamily comets form a population broadly analogous to the Oort cloud as perceived by a planet orbiting the sun. There are differences. The JFCs are not in general bound to Jupiter, so that in general their orbits are hyperbolic, while the Oort cloud is bound to the Sun in orbits that are very nearly parabolic. But there are many points of similarity. Even for the hyperbolic orbits, encounter velocites are rarely large compared to orbital velocities of the satellites (e.g., $v_{\infty} \approx 5 \mathrm{~km} / \mathrm{s}$ at Jupiter, vs $v_{\text {orb }}=10.9 \mathrm{~km} / \mathrm{s}$ for Ganymede). Moreover, many of the comets that hit Jupiter do so while in orbit around Jupiter. Shoemaker-Levy 9 provides an example. KD96 found that $15 \%$ of the comets that hit Jupiter in their simulation were temporary satellites; we find that 30 of the 144 objects to hit Jupiter (20\%) 


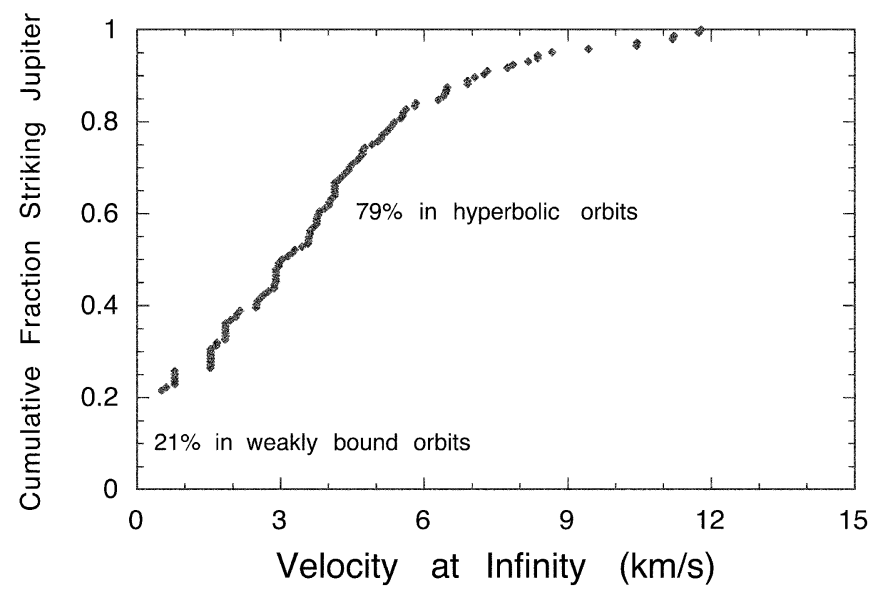

FIG. 1. The cumulative distribution of encounter velocities $\left(v_{\infty}\right)$ with Jupiter (velocities at infinity) for the 144 objects striking Jupiter in LD97. The objects plotted at $v_{\infty}=0$ were orbiting Jupiter when they struck the planet; these are $21 \%$ of the total.

in the LD97 simulation did so from weakly bound (nearly parabolic) orbits. Encounter velocities with Jupiter (velocities at "infinity") for these 144 objects are shown in Fig. 1.

As with Oort cloud comets encountering Earth, we expect orbital inclinations to be nearly isotropic with respect to the plane of satellite orbits. The reason is that, like the Kuiper Belt, the JFC population is better pictured as a thick torus than as a thin disk. Because the torus is thick compared to Jupiter's Hill sphere (the sphere within which Jupiter's gravity is more important than the Sun's), inclinations will be more or less random; and because on average we expect as many comets to be near perihelion while encountering Jupiter as near aphelion, we do not expect there to be a significant azimuthal asymmetry. Figure 2

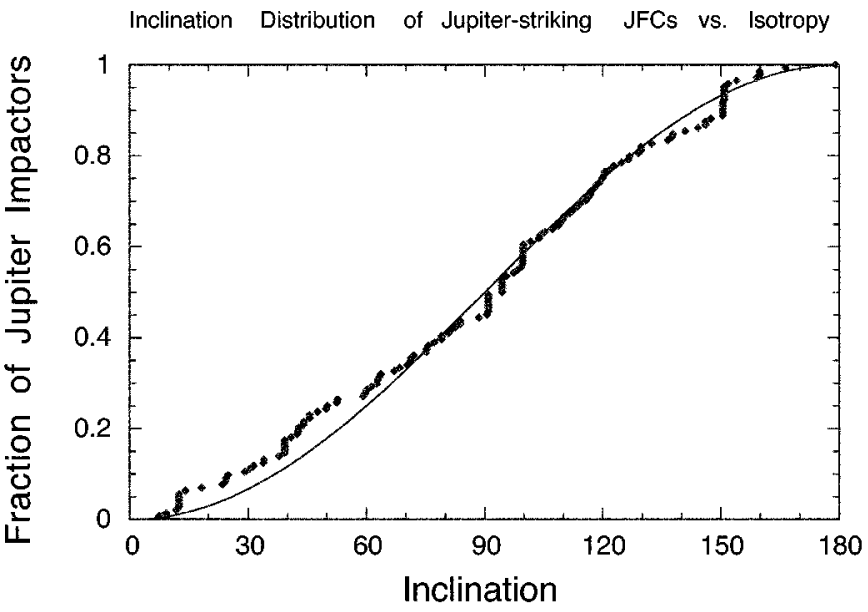

FIG. 2. The cumulative jovicentric inclination distribution of the 144 test particles that hit Jupiter in LD97. This is compared to the isotropic distribution. compares the inclination distribution of the 144 test particles that hit Jupiter in LD97 to the isotropic distribution. There is a modest excess of low inclination encounters among the test particles, but the influence of this slight asymmetry on the relative cratering rates at Jupiter is negligible compared to other uncertainties.

Here we will calculate impact probabilities and impact velocities using Öpik's formulae as written for hyperbolic orbits (SW82). We have used them in the following form (satellite orbits are assumed circular):

$$
\begin{gathered}
U^{2}=3-(1-e) / q-2 \cos i \sqrt{q(1+e)} \\
U_{\mathrm{x}}^{2}=2-(1-e) / q-q(1+e) \\
v_{\mathrm{i}}^{2}=v_{\mathrm{esc}}^{2}+v_{\mathrm{orb}}^{2} U^{2} .
\end{gathered}
$$

$U$ and $U_{\mathrm{x}}$ are the encounter velocity and the radial component of the encounter velocity in units of the satellite's orbital velocity $v_{\text {orb }} ; v_{\mathrm{i}}$ and $v_{\text {esc }}$ are the impact and escape velocities; $e$ and $q$ are the jovicentric eccentricity and perijove of the comet's orbit. The perijove distance $q$ is expressed in units of the semimajor axis $a_{\text {sat }}$ of the satellite's orbit; impacts are possible if and only if $q \leq 1$. The escape velocity $v_{\text {esc }}$ is that at the surface of the satellite. The impact probability per orbit is

$$
P_{\mathrm{i}}=\frac{R_{\mathrm{sat}}^{2}}{a_{\mathrm{sat}}^{2}}\left(1+\frac{v_{\mathrm{esc}}^{2}}{v_{\mathrm{orb}}^{2} U^{2}}\right) \frac{U}{U_{\mathrm{x}}} \frac{1}{\pi \sin i},
$$

where $R_{\text {sat }}$ is the satellite's radius.

For a given satellite $R_{\text {sat }}, a_{\text {sat }}, v_{\text {esc }}$, and $v_{\text {orb }}$ are known. A stray comet is described by $i, q$, and $e$. Thus, for each simulated comet, the triad $(i, q, e)$ is generated with three random numbers. These in turn determine impact probabilities and impact velocities for each comet in the Monte Carlo simulation.

Inclinations. We will assume isotropic inclinations. The normalized cumulative distribution is

$$
N(<i)=\frac{1-\cos i}{2}
$$

where the notation $N(<i)$ refers to the cumulative number with inclinations less than $i$. In a Monte Carlo simulation, a random number $0<x<1$ is identified with the normalized cumulative distribution; hence,

$$
\cos i(x)=1-2 x
$$

We have also performed simulations using the inclination distribution shown in Fig. 2, but our results are insensitive to the differences between the LD97 distribution and isotropy. 
Perijove. For a comet to strike a satellite, it is necessary that $q \leq 1$. If $q<q^{\prime} \equiv R_{\mathrm{J}} / a_{\text {sat }}$, the comet strikes Jupiter. There are simple expressions for perijove distributions in the limits of low and high energy encounters. It is well known that the perihelion distribution of parabolic comets on their first visit to the inner solar system should be uniform; i.e., the cumulative distribution is $N(<q) \propto q$. The same applies to JFCs in nearly parabolic orbits encountering Jupiter. In the high velocity limit, where Jupiter's gravity can be neglected, encounters are uniformly distributed in area (i.e., Jupiter is at the center of a disk that is uniformly struck). Thus $N(<q) \propto q^{2}$ for strongly hyperbolic orbits.

In the general case one begins with the impact parameter $b$, the perijove distance in the absence of gravitational focusing, for which $N(<b) \propto b^{2}$. From energy and angular momentum conservation the general expression for $q(b)$ is

$$
q=\frac{v_{\mathrm{orb}}^{2}}{v_{\infty}^{2}}\left(\sqrt{1+b^{2} v_{\infty}^{4} / v_{\mathrm{orb}}^{4}}-1\right)
$$

where both $b$ and $q$ are in units of $a_{\text {sat }}$. For $0<q<1$, $0<b^{2}<1+2 v_{\text {orb }}^{2} / v_{\infty}^{2}$. As $b^{2}$ is uniform, we generate random values of $q$ from random numbers $0<x<1$ by

$$
b^{2}=x\left(1+2 v_{\mathrm{orb}}^{2} / v_{\infty}^{2}\right)
$$

and insert this value of $b^{2}$ in Eq. (8) to get $q(x)$. We treat the weakly bound orbits separately as parabolae, for which $e=1$ and $N(<q)=q=x$.

Eccentricity. The eccentricity of a hyperbolic orbit is greater than one. In terms of the comet's velocity $v_{\infty}$ at infinity, the eccentricity is

$$
e=1+q v_{\infty}^{2} / v_{\text {orb }}^{2}
$$

The perijove distance $q$ is given in terms of the satellite's semimajor axis $a_{\text {sat }}$. Typical $v_{\infty}$ for JFCs is $\sim 5 \mathrm{~km} / \mathrm{s}$, i.e., about $\sqrt{3-T} \approx 0.4$ of Jupiter's orbital velocity. Because $v_{\infty}^{2}<v_{\text {orb }}^{2}$ for all of the Galilean satellites, our results are insensitive to $v_{\infty}$. (Recall that we are not attempting to calculate the absolute impact rate, which would be sensitive to $v_{\infty}$. Rather we are calculating the impact rate on a satellite relative to the impact rate on Jupiter.)

To first approximation, the velocity distribution shown in Fig. 1 is adequate to describe $v_{\infty}$ pertinent to encounters with any of the Galilean satellites. This works because relatively few orbits are strongly hyperbolic (i.e., few have $v_{\infty}^{2} \gg v_{\text {ort }}^{2}$ ). The number of objects crossing a satellite's orbit then scales as $1 / q^{\prime}=a_{\mathrm{sat}} / R_{\mathrm{J}}$. For example, 144 hits on Jupiter would correspond to 850 Io-crossers. A better approximation can be obtained using Eqs. (8) and (9), solving

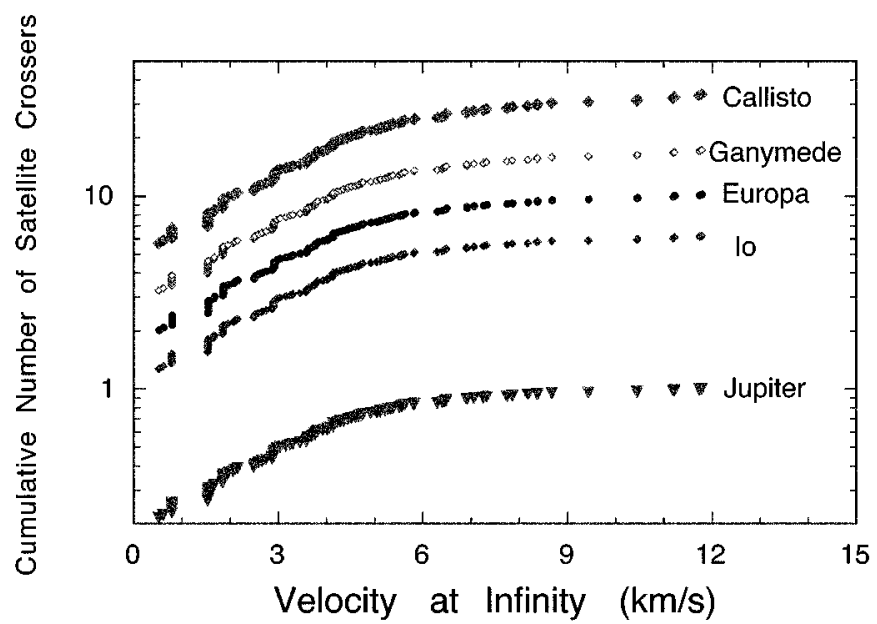

FIG. 3. Cumulative $v_{\infty}$ distributions for JFCs hitting Jupiter (normalized to unity) and the relative number crossing the orbits of the Galilean satellites. The fraction in orbit around Jupiter ranges from $21 \%$ of those hitting Jupiter to $4 \%$ of those passing Callisto.

for $x$. The number of objects with $q \leq 1$ scales from the number of objects with $q \leq q^{\prime}$ as

$$
N(q \leq 1)=N\left(q \leq q^{\prime}\right) \frac{v_{\infty}^{2}+2 v_{\mathrm{orb}}^{2}}{v_{\infty}^{2} q^{\prime 2}+2 v_{\mathrm{orb}}^{2} q^{\prime}} .
$$

The scaling is a function of $v_{\infty}$. The normalized $v_{\infty}$ distributions that result for Jupiter and the different satellites are shown in Fig. 3. Objects with smaller encounter velocities are progressively more important for the closer satellites, as these objects are the most strongly focused gravitationally. Only 4\% of the JFCs crossing Callisto's orbit are in weakly bound orbits vs $17 \%$ at Io. On the other hand, the number of comets crossing Callisto's orbit is larger-35 comets pass Callisto for every one that hits Jupiter.

Impact velocity distributions for the simulated JFCs at the various satellites are shown in Fig. 4. Satellite parameters, impact probabilities relative to impact on Jupiter, average impact velocities, and a fair amount of other material to be discussed below, are listed in Table I. NY95 also estimated relative impact probabilities on the satellites and Jupiter, but they do not use Öpik's equations. Rather, they use approximate expressions of their own devise. Impact rates relative to impacts on Jupiter are roughly $60 \%$ of what we find. The source of this difference is unclear.

\subsection{Crater Diameter}

We use the expression

$$
D_{\mathrm{s}}=1.65\left(\frac{m_{\mathrm{i}}}{2 \rho_{\mathrm{t}}}\right)^{0.26} g^{-0.22} v_{\mathrm{i}}^{0.44}\left(\frac{\rho_{\mathrm{i}}}{\rho_{\mathrm{t}}}\right)^{0.073}(\cos \theta)^{0.44} \mathrm{~cm}
$$




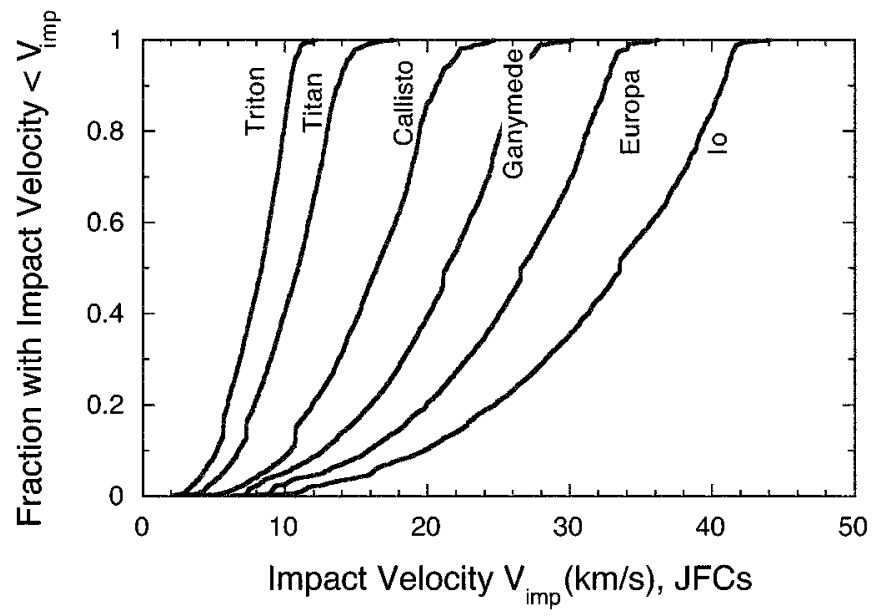

FIG. 4. Cumulative impact velocity distributions for impacts by ecliptic comets on various satellites in the solar system. These are the result of a Monte Carlo simulation; the occasional unsightly glitches are caused by rare objects in orbits with very high impact probability. for the diameter of a simple crater given impactor mass $m_{\mathrm{i}}$, impact velocity $v_{\mathrm{i}}$, incidence angle $\theta$ measured from the vertical, surface gravity $g$, and impactor and target densities $\rho_{\mathrm{i}}$ and $\rho_{\mathrm{t}}$. It is to be evaluated in cgs units. The appearance of three significant digits is an illusion; the leading factor is better regarded as being between 1.3 and 2. Equation (12) is essentially the expression recommended by Schmidt and Housen (1987). Crater efficiency (the proportionality constant in Eq. (12)) is problematic and necessarily introduces considerable uncertainty into our calculations. The uncertainty in the cratering efficiency is probably about a factor of two in crater volume. The uncertainty in crater diameter for a given impactor is probably about $30 \%$, which translates to a factor two uncertainty in impactor flux.

We have appended the term to Eq. (12) that allows for incidence angle. To first approximation, it appears that only the normal component of the impact velocity contributes to cratering (e.g., Chapman and McKinnon 1986, p. 507). The median value of $(\cos \theta)^{0.44}$ is $\left(\cos 45^{\circ}\right)^{0.44}=$ 0.86 . Other scalings with incidence angle (e.g., $(\cos \theta)^{0.33}$

TABLE I

Jupiter Family Comets

\begin{tabular}{|c|c|c|c|c|c|c|}
\hline & Earth & Jupiter & Io & Europa & Ganymede & Callisto \\
\hline$R_{\mathrm{sat}}{ }^{a}$ & 6380 & 71400 & 1820 & 1570 & 2630 & 2400 \\
\hline$a_{\mathrm{sat}} / R_{\mathrm{J}}^{b}$ & - & - & 5.9 & 9.4 & 15.0 & 26.4 \\
\hline$v_{\text {orb }}^{c}$ & 29.7 & 13.0 & 17.3 & 13.7 & 10.9 & 8.2 \\
\hline$g^{d}$ & 981 & 2490 & 181 & 131 & 143 & 125 \\
\hline$\rho_{\mathrm{t}}^{e}$ & 2.8 & & 2.8 & 1.0 & 1.0 & 1.0 \\
\hline$N_{\text {sat }} / N_{\text {Jup }}{ }^{f}$ & - & 1 & 6.2 & 10.3 & 17 & 34 \\
\hline$f_{\text {orb }}{ }^{g}$ & - & 0.21 & 0.17 & 0.13 & 0.08 & 0.04 \\
\hline$\left\langle v_{\mathrm{i}}\right\rangle^{h}$ & 22 & 60 & 32 & 26 & 21 & 16 \\
\hline$P_{\mathrm{i}, \mathrm{rel}}^{i}$ & $3.1 \times 10^{-5}$ & 1.0 & $1.4 \times 10^{-4}$ & $6.2 \times 10^{-5}$ & $1.2 \times 10^{-4}$ & $6.1 \times 10^{-5}$ \\
\hline$P_{\mathrm{i}}(d>1)^{j}$ & $3.5 \times 10^{-7}$ & 0.011 & $1.5 \times 10^{-6}$ & $7.0 \times 10^{-7}$ & $1.4 \times 10^{-6}$ & $6.9 \times 10^{-7}$ \\
\hline$D_{\mathrm{c}}{ }^{k}$ & 4 & - & 15 & 4 & 4 & 4 \\
\hline$m_{\mathrm{i}}(D=20)^{l}$ & $1.1 \times 10^{16}$ & - & $2.6 \times 10^{15}$ & $4.1 \times 10^{14}$ & $6.4 \times 10^{14}$ & $9.1 \times 10^{14}$ \\
\hline$d_{\mathrm{i}}(D=20)^{m}$ & 3.0 & - & 1.83 & 1.0 & 1.15 & 1.29 \\
\hline$\tau(D>20)^{n}$ & 34 & - & 2.5 & 1.4 & 1.0 & 2.6 \\
\hline$\dot{C}(D>10)^{\circ}$ & $3.0 \times 10^{-16}$ & - & $4.5 \times 10^{-14}$ & $1.0 \times 10^{-13}$ & $5.4 \times 10^{-14}$ & $2.5 \times 10^{-14}$ \\
\hline
\end{tabular}

${ }^{a}$ Radius [km].

${ }^{b}$ Semimajor axis [jovian radii].

${ }^{c}$ Circular orbital velocity $[\mathrm{km} / \mathrm{s}]$.

${ }^{d}$ Surface gravity $\left[\mathrm{cm} / \mathrm{s}^{2}\right]$.

${ }^{e}$ Presumed crustal density $\left[\mathrm{g} / \mathrm{cm}^{3}\right]$.

${ }^{f}$ Relative number of comets in satellite-crossing orbits.

${ }^{g}$ Fraction of these in weakly bound orbits.

${ }^{h}$ Average impact velocity $[\mathrm{km} / \mathrm{s}]$.

${ }^{i}$ Relative impact rate normalized to impacts on Jupiter.

${ }^{j}$ Impact frequency, $d>1 \mathrm{~km}\left[\mathrm{yr}^{-1}\right]$.

${ }^{k}$ Transition to complex crater, diameter [km].

${ }^{l}$ Impactor mass [g] giving $D=20 \mathrm{~km}$ crater at $\theta=45^{\circ}$.

${ }^{m}$ Corresponding impactor diameter $[\mathrm{km}]$ at $\rho_{\mathrm{i}}=0.8 \mathrm{~g} / \mathrm{cm}^{3}$.

${ }^{n}$ Timescale [Ma] for $D>20 \mathrm{~km}$ craters.

${ }^{o}$ Cratering rate, $D>10 \mathrm{~km}\left[\mathrm{~km}^{-2} \mathrm{yr}^{-1}\right]$. 
(Melosh 1989 p. 121), and $(\cos \theta)^{0.67}$ (Shoemaker et al. 1990)) are in use, with median values ranging from 0.79 to 0.89 , but to simplify our discussion of apex-antapex asymmetries (below) we will scale with the normal component of the impact velocity.

Larger craters are shallower, wider, and considerably more complicated than the simple bowl-shaped crater described by Eq. (12). The final crater diameter is parameterized for $D_{\mathrm{s}}>D_{\mathrm{c}}$ by

$$
D_{\mathrm{f}}=D_{\mathrm{s}}^{1.13} D_{\mathrm{c}}^{-0.13},
$$

where $D_{\mathrm{c}}$ marks the transition between simple and complex craters (McKinnon et al. 1991). We have used $D_{\mathrm{c}}=4$ $\mathrm{km}$, which is the value recommended by Chapman and McKinnon (1986) for Ganymede. Therefore for complex craters we have effectively

$$
D_{\mathrm{f}} \propto m_{\mathrm{i}}^{0.294}\left(v_{\mathrm{i}} \cos \theta\right)^{0.5} .
$$

The dependence on mass is exactly that used by SW82 and Shoemaker et al. (1990). Although we will use Eq. (14) as though it were true, the power law exponent in Eq. (13) is quite uncertain. Moreover, as complex craters are not self-similar, one suspects that the collapse of the transient crater does not obey a simple power law, but rather a more complicated function that will need to be deduced empirically.

It is useful, albeit a little misleading, to relate crater diameter directly to comet diameter. Table I lists impactor masses that give $20-\mathrm{km}$ diameter craters on the various Galilean satellites, at the average impact velocities (Table I), at the median (and most probable) incidence angle $\left(45^{\circ}\right)$, into icy or rocky target material (assumed densities also listed in Table I). Diameters of corresponding $\rho=0.8$ $\mathrm{g} / \mathrm{cm}^{3}$ density comets are also listed. Craters are wider on the icy moons than on Io because it is the mass of excavated material that is important, not the volume. Twenty-kilometer craters are made by kilometer-size comets. Because crater diameter scales like $D \propto d^{0.83 \pm 0.05}$, it takes a 200 -m object to make a $5-\mathrm{km}$ crater. Other than the short lived pieces of SL9, such small comets are not yet known to exist. At the other extreme, the relatively young ganymedean basin Gilgamesh, 600-km diameter, is the product of a $\sim 50-\mathrm{km}$ diameter comet.

\subsection{Calibration to JFCs in the Inner Solar System}

The masses and numbers of JFCs are the greatest source of uncertainty in the Galilean cratering rates. Masses of active comets are hard to assess because the nuclei are wrapped in fog, while the number is hard to assess because it appears that most JFCs are inactive. Calibration involves four issues that relate what we see near Earth to what is perceived at Jupiter: (i) the spatial distribution of JFCs; (ii) the shape of their mass distribution; (iii) the relative importance of inactive comets; and (iv) the absolute mass of a comet given some measure of its brightness. The last is the least certain. Although the issues are separate they are not separable.

SW82 and Shoemaker et al. (1994; hereafter SWS94) use Roemer's photographic $B(1,0)$ magnitudes to estimate the magnitudes of cometary nuclei (Roemer et al. 1966). SW82 argued that there are 40 active JFCs for which $q<1.7$ AU and $B(1,0)<16$, and that this sample is effectively complete; i.e., they take $N(q<1.7, B(1,0)<16)=40$. According to SW82, at $B(1,0)=16$ a cometary nucleus of albedo 0.03 would have a diameter of $6.3 \mathrm{~km}$. This assumption of extremely low albedo has been mostly borne out by subsequent observation. However, when SW82 applied this scaling to long period comets they calculated impact rates at Earth that were too high. Therefore they arbitrarily corrected $B(1,0)$ for all active comets by 2.3 magnitudes. The correction presumes that these comets are too bright because all retained comae when observed. The revised diameter at $B(1,0)=16$ is therefore $2.2 \mathrm{~km}$, and so effectively SW82 calibrate the number of active JFCs to $N(q<1.7, d>2.2)=40$.

For the size distribution SW82 take

$$
N(>d) \propto d^{-1.97} \rightarrow N(>m) \propto m^{-0.66} .
$$

Equation (15) is consistent both with the observed distribution of $B(1,0)$ and with the number of bright-rayed craters on Ganymede, for which $N(>D) \propto D^{-2.2}$ for $25<D<$ $100 \mathrm{~km}$ diameter (Passey and Shoemaker 1982). Using $D \propto m^{-0.294}$ (see above), one deduces that $N(>m) \propto m^{-0.65}$. Theory tells us that a fragmentation cascade evolves towards a $N(>d) \propto d^{-2.5} \propto m^{-0.833}$ power law distribution (Dohnanyi 1972, Safronov 1972, Williams and Wetherill 1994, Tanaka et al. 1996, Durda and Dermott 1997) that is somewhat richer in small objects than Shoemaker's distribution. The distribution of active absolute magnitudes $H_{10}$ is also claimed to be consistent with $N(>m) \propto m^{-0.7}$ (Donnison 1986, Hughes 1988), although this is controversial (see Weissman 1990 for a different point of view) and considerably more uncertain, in view of the dubious mapping of $H_{10}$ onto $m$ (a topic we will address below). In our opinion, the steeper theoretical distribution is a good guess for the mass distribution of new comets; it is also reasonable to presume that smaller comets are more subject to thermal disintegration than larger comets, so that the mass distribution might tend to flatten as the ensemble ages. For specificity we will use Shoemaker's distribution.

The extrapolation to $1-\mathrm{km}$ comets is plausible. For one thing, the observed crater distribution extends to $25-\mathrm{km}$ craters, which would be made by kilometer-size comets. Such comets are reasonably likely to exist in large numbers: 
based on an assumed radar albedo of 0.039 , Hyakutake (an LPC) is estimated to have been $2-3 \mathrm{~km}$ diameter (Harmon et al. 1997) and Sugano-Saigusa-Fujikawa less than $1 \mathrm{~km}$ (Harman et al. 1997); the parent of SL9 was about 1.5- to 1.8-km diameter (Asphaug and Benz 1996); and according to Brandt et al. (1997), HST observations indicate that the nuclei of Honda-Mrkos-Pajdusakova and Wirtanen (Lamy et al. 1998) are no greater than 0.7- and $1.2-\mathrm{km}$ diameter, respectively. Brandt et al. (Lamy et al. 1998) argue that Shoemaker's $N(>d) \propto d^{-2}$ power law holds to $200 \mathrm{~m}$. Nevertheless, extrapolation to much smaller comets and smaller craters is somewhat dangerous, and we are not entirely comfortable extrapolating to the 20 -m comets required to make $1-\mathrm{km}$ craters.

That extinct JFCs would be important was emphasized by SW82, but their importance was amplified by subsequent work. SWS94 considered the discovery rate of extinct comets and from this estimated that the ratio of extinct to active comets with $q<2 \mathrm{AU}$ is roughly 20 and that the ratio of impacts on Earth by extinct comets to those by active comets is roughly $14 .^{1}$

SWS94 also estimate that there are 40 active JFCs with $q<1.0$ AU and $d>1 \mathrm{~km}$; i.e., $N(q<1, d>1)=40$. At an average impact rate of $1.3 \times 10^{-9}$ per year per comet, and multiplying by fourteen to include inactive comets, SWS94 get $\dot{N}_{\oplus}(d>1)=7 \times 10^{-7} \mathrm{yr}^{-1}$ for the rate JFCs (active and inactive) strike Earth. The argument for 40 active comets begins with a table listing 13 active Earthcrossing JFCs, states that only 8 of 17 IRAS dust bands have been associated with known comets, and that therefore there are really 25-40 active Earth-crossing JFCs. But in fact there are presently only five or six Earth-crossing JFCs: $\mathrm{P} /$ Encke is not a JFC; $\mathrm{P} / \mathrm{Biela}$ is disintegral, and four others are single apparition objects or lost; and $\mathrm{P} /$ Tuttle and probably P/Machholz 1 are Halley-type comets (Bailey and Emel'yanenko 1996). Most known JFCs with $q<1$ were discovered in the 18th and 19th centuries, a discovery history quite unlike that of the more distant JFCs (Fernandez et al. 1992). Thus Fernandez et al. suggested that the count is nearly complete. On closer inspection, the IRAS data supports Fernandez et al. There were actually 103 IRAS dust bands, 94 of which are attributed to eight previously known comets (Sykes and Walker 1992). Seven of the eight comets were themselves seen in the IRAS data (P/Encke, then far from perihelion, was the eighth). Of the nine unattributed dust bands, eight are faint. Four are

\footnotetext{
${ }^{1}$ The argument places a fair amount of weight on Hidalgo, an object with $q=1.969 \mathrm{AU}, T=2.07$, and $B(1,0)=11.6$. If it has albedo of $3 \%$ and no coma, its diameter would be $50 \mathrm{~km}$. SWS94 assume that Hidalgo is an extinct JFC, and take it as representative of the largest nearby member of its class. However, with $T=2.07$ Hidalgo is arguably not even a JFC; it is almost as likely to be an extinct HTC. But no matter; at least a score of likely extinct JFCs have since been discovered, including one nearly as large as Hidalgo.
}

at high ecliptic latitudes and so are not plausibly associated with JFCs. The fraction of unattributed JFC dust bands is therefore more like five of 99 . The brightest of the nine is associated with the coma of a previously unknown comet. This would imply that the number of nearby undiscovered active JFCs is one in eight (the fraction of previously unknown observed comets associated with the dust bands). The other four unidentified bands are as likely to have been abandoned by changing orbits of known JFCs as not (Sykes and Walker 1992). In sum, the case for 40 active Earth-crossing JFCs is not compelling. The true number is probably closer to ten, and so the current impact rate at Earth would be closer to $\dot{N}_{\oplus}(d>1)=2 \times 10^{-7} \mathrm{yr}^{-1}$. However, this estimate is strongly affected by small number statistics.

Levison and Duncan (LD97) give relative impact rates that apply to the entire population of JFCs, active and inactive. The total number of comets, active and inactive, is obtained by using a model with an effective "fade time" for JFCs; the best fit to the observed orbital distribution of JFCs is for a fade time of 12,000 years (older JFCs are treated as extinct). LD97 also find that, for the population of JFCs that make at least one perihelion passage with $q<2.5 \mathrm{AU}$, the ratio of extinct to active comets is about 3.5. This ratio refers to the population as a whole; the ratio of extinct to active comets appears to be higher near the Earth and is not necessarily inconsistent with SWS94's estimate.

LD97 calibrate the number of active comets in their model to Fernandez et al.'s estimate of the number of bright, active JFCs with perihelia less than 2.0 AU. Fernandez et al. (1992) argued that the number of active JFCs with $q<1.5 \mathrm{AU}$ and $H_{\mathrm{T}}<9$ is 12 and that the discovery history indicates that this is basically complete; they extrapolate this to estimate that $N\left(q<2, H_{\mathrm{T}}<9\right)=40$. For the impact rate at Earth, LD97 find

$$
\dot{N}_{\oplus}\left(H_{\mathrm{T}}<9\right)=7.5 \times 10^{-8} \mathrm{yr}^{-1},
$$

and for the impact rate on Jupiter,

$$
\dot{N}_{\mathrm{J}}\left(H_{\mathrm{T}}<9\right)=2.4 \times 10^{-3} \mathrm{yr}^{-1} .
$$

LD97 take $H_{\mathrm{T}}=9$ to mean $2_{-1}^{+2} \mathrm{~km}$ diameter, thus $\dot{N}_{\mathrm{J}}(d>$ 1) $=9.6 \times 10^{-3} \mathrm{yr}^{-1}$ to a factor of four.

Our purpose here is to use S82's estimate of $40 \mathrm{JFCs}$ with $q<1.7$ AU and $d>2.2 \mathrm{~km}$ to get impact rates of $1-\mathrm{km}$ comets on Earth and Jupiter. SW82 extrapolated the perihelion distribution by assuming that it is uniform beyond 1.3 AU. We will replace this by calibrating SW82's 40 comets with $N(q<1.7)$ to the LD97 simulation. The latter has the advantages of offering better and unbiased statistics. LD97's calibration is equivalent to $N(q<1.7)=$ 23 active comets. Therefore the recalibrated impact rate 
on Earth is $\dot{N}_{\oplus}(>d)=40 / 23 \times 7.5 \times 10^{-8}(d / 2.2 \mathrm{~km})^{-1.97}=$ $6.2 \times 10^{-7}(d / 1 \mathrm{~km})^{-1.97} \mathrm{yr}^{-1}$. By coincidence, this is virtually identical to SWS94's published estimate. The corresponding impact rate on Jupiter is

$$
\begin{aligned}
\dot{N}_{\mathrm{J}}(>d) & =40 / 23 \times 2.4 \times 10^{-3}(d / 2.2 \mathrm{~km})^{-1.97} \\
& =0.020(d / 1 \mathrm{~km})^{-1.97} \mathrm{yr}^{-1}
\end{aligned}
$$

This rate is a bit higher than the others we will consider, but it is reasonable. According to Eq. (18), a comet as large as SL9 strikes Jupiter every 100-150 years.

An independent calibration is based on the presumptive relationship between the absolute magnitude $H_{10}$ or $H_{\mathrm{T}}$ of an active comet and its diameter $d$ or mass $m$ (e.g., Weissman 1990, Fernandez et al. 1992, Bailey et al. 1994, NY95). The advantage of this approach is that it represents the consensus judgement of experts in the field. The two versions most often quoted are (e.g., Weissman 1990, Rahe et al. 1994)

$$
\log _{10}(m)=20.0-0.4 H_{10}
$$

and

$$
\log _{10}(m)=19.9-0.5 H_{10}
$$

For kilometer-size comets these differ by an order of magnitude. In these expressions $H_{10}$ refers to a particular list cometary absolute active magnitudes; a somewhat different definition of absolute magnitude, $H_{\mathrm{T}}$, yields another list (Kresak and Kresakova 1989). The two versions of absolute magnitude are quite different, with $H_{\mathrm{T}}$ being on average about 1.9 magnitudes brighter than $H_{10}$, with a scatter of 1.5 magnitudes (Kresak and Kresakova 1989).

At best, expressions like Eqs. (19) and (20) can only be broad statistical relations; they are effectively worthless when applied to individual comets (see Kary and Dones 1996 for a more thorough discussion). To illustrate, consider two examples: Hyakutake was a 2- to 3-km-size comet with $H \approx 5$, i.e., it had roughly the same absolute magnitude as $\mathrm{P} / \mathrm{Halley}$, a $9-\mathrm{km}$ object. The second example is IRASAraki-Alcock, an unimpressive visual sight $(H>10)$ that passed only 0.031 AU from Earth in 1983 but, as measured by radar, is almost as big as P/Halley. In other words, the intrinsic scatter in masses computed using a relation like Eq. (19) is at least a factor of $10^{4}$. The matter is made worse by multiple and arbitrary definitions of cometary magnitude.

According to Eqs. (19) and (20), a comet with $H_{10}=$ 10.9 (corresponding to $H_{\mathrm{T}}=9$ ) would have a mass of $8 \times$ $10^{15} \mathrm{~g}$ or $8 \times 10^{14} \mathrm{~g}$, respectively. For $N(>m) \propto m^{-0.66}$ and $\rho=0.8 \mathrm{~g} / \mathrm{cm}^{3}$, the corresponding impact rates of $1-\mathrm{km}$ comets at Jupiter $\dot{N}_{\mathrm{J}}(d>1)$ are 0.012 and $0.003 \mathrm{yr}^{-1}$, respec- tively. The former is essentially the same as the other rates we have deduced here, while the latter is somewhat smaller. Whether one should take relations like Eqs. (19) and (20) seriously is an open issue.

A third approach (Kary and Dones 1996) uses only nuclear sizes obtained by observing relatively large, relatively inactive comets for which there is corrollary evidencesuch as a reproducible rotational light curve-to indicate that one is actually seeing the nucleus (Jewitt 1991). Short of going there this is the best way to measure a comet; unfortunately there are very few reliable sizes available, and these are probably biased toward large, relatively inactive objects that might be less representative than one would hope.

There are three active JFCs with diameters $\geq 10 \mathrm{~km}$ and perihelia inside $2 \mathrm{AU}$ (all three are Mars-crossers): P/ Neujmin 1, P/Tempel 2, and P/Arend-Rigaux. The list is probably complete. $\mathrm{P} /$ Neujmin 1 , at $d \approx 20 \mathrm{~km}$, is clearly big. Jewitt (1991) estimated that the other two have $d \approx$ $10 \mathrm{~km}$. Fernandez et al. (1992) provided a longer list of nuclear magnitudes; according to their list $\mathrm{P} / \mathrm{Tempel} 2$ has $d=12 \mathrm{~km}$ and $\mathrm{P} /$ Arend-Rigaux has $d=8 \mathrm{~km}(\mathrm{P} /$ Neujmin 1 waddles in at $22 \mathrm{~km}$ ). Kary and Dones (1996) based their estimate of the number of active JFCs on the assumption that $N(q<2.5, d>9.7)=5 \pm 2$. It is noteworthy that $\mathrm{P} /$ Neujmin 1 and P/Arend-Rigaux are very nearly inactive (Kronk 1984). We will take $N(q<2, d>10)=2 \pm 1$. When this is extrapolated to $1-\mathrm{km}$ diameter using $N(>d) \propto$ $d^{-1.97}$, we obtain

$$
N(q<2,>d)=200 \pm 100(d / 1)^{-1.97}
$$

active comets. This is then calibrated to LD97, for which there are $N(q<2)=40$ active comets, to give total impact rates of kilometer-size comets (active and inactive) at Earth and Jupiter of

$$
\begin{aligned}
\dot{N}_{\oplus}(d>1) & =4 \pm 2 \times 10^{-7} \mathrm{yr}^{-1} \text { and } \\
\dot{N}_{\mathrm{J}}(d>1) & =0.012 \pm 0.006 \mathrm{yr}^{-1}
\end{aligned}
$$

respectively. These rates are essentially identical to the recommended rates we quote in Table I. If we were to regard $\mathrm{P} /$ Neujmin 1 and $\mathrm{P} /$ Arend-Rigaux as effectively inactive (i.e., discovered only because they are big), we would have $N(q<2, d>10)=1 \pm 1$, for which $\dot{N}_{\mathrm{J}}(d>$ $1)=0.006 \pm 0.006 \mathrm{yr}^{-1}$. On the other hand, the single power law exponent we would favor from strictly theoretical concerns, $N(<d) \propto d^{-2.5}$, were we to use it here, would raise the latter estimate to $\dot{N}_{\mathrm{J}}(d>1)=0.02 \pm 0.02 \mathrm{yr}^{-1}$. The uncertainty is perforce large.

\subsection{Calibration to Observed Encounters with Jupiter}

In principle the best way to estimate the number of comets encountering Jupiter is by counting the number of 


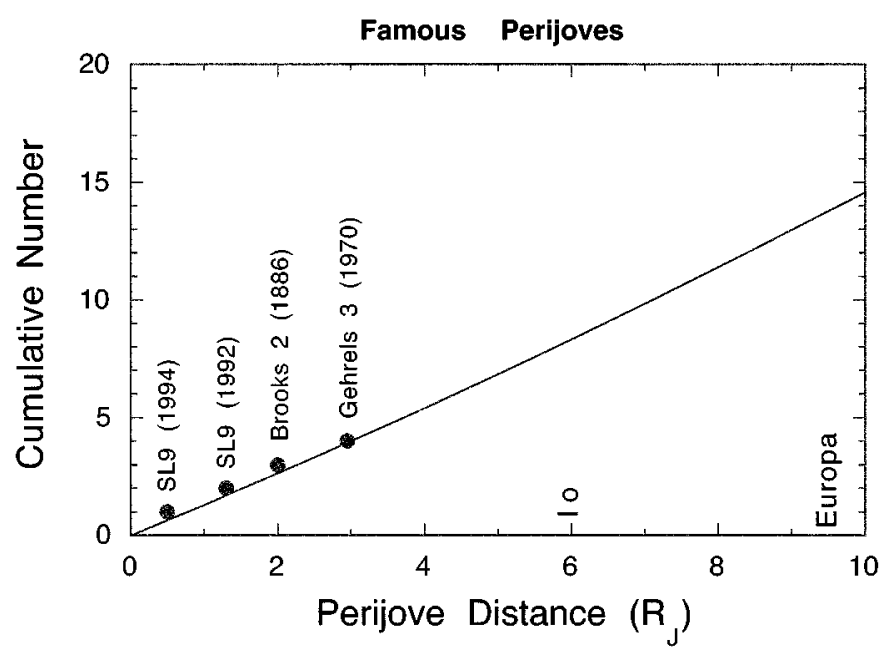

FIG. 5. Cumulative plot of known close perijoves; the name of the comet and the year of perijove are indictated. The curve is extrapolated to Europa's orbit using Eq. (11) and an average $v_{\infty}=5 \mathrm{~km} / \mathrm{s}$. The four close approaches in the last 120 years imply eight Io-crossers and 14 Europa crossers in the same period.

comets encountering Jupiter. Three comets - all JFCSare known to have made four close approaches to Jupiter in the past 150 years (Fig. 5).

The most famous of these is D/Shoemaker-Levy 9, which passed $1.3 \mathrm{R}_{\mathrm{J}}$ above the center of Jupiter in 1992, there to be torn apart by tides, and then would have passed perijove at $0.5 \mathrm{R}_{\mathrm{J}}$ in 1994 had Jupiter not intervened. According to KD96, the successive close approaches should be regarded as uncorrelated, in the sense that most (98\%) comets in orbit about Jupiter do not make two successive close passes.

It is the other two comets that give us statistics. In 1970, $\mathrm{P} /$ Gehrels 3 passed perijove at $3.0 R_{\mathrm{J}}$. This comet was discovered in 1975. It does not appear to have been tidally disrupted. The other is more interesting. $\mathrm{P} /$ Brooks 2 passed perijove at $2.0 R_{\mathrm{J}}$ in 1886 . As a consequence of its close encounter with Jupiter it was tossed into the inner solar system, where it was discovered approaching perihelion at $\sim 1.9$ AU in 1889 . It was fairly bright $\left(m_{\mathrm{v}} \approx 8\right)$, but more peculiar is that it consisted of at least five unequal pieces. The chief fragment of P/Brooks 2 has been consistently recovered during favorable apparitions for the past century. It has faded more or less monotonically since, and is now at least six magnitudes fainter than in 1889 (Sekanina and Yeomans 1985).

Apart from their interactions with Jupiter, none of these comets seem unusual. In particular, all were large enough to have left $15-$ to $30-\mathrm{km}$ diameter craters on Europa. As mentioned above, D/SL9 was originally $1.5-1.8 \mathrm{~km}$ (1992), and a few of its 1994 fragments were of order 1-km diameter. The surviving remnant of $\mathrm{P} /$ Brooks 2 has been esti- mated at $0.8 \mathrm{~km}$ (Sekanina and Yeomans 1985). Barucci et al. (1996) found that P/Gehrels 3 has a spectrum like a D-type asteroid. According to Scotti (1998), P/Gehrels 3 had nuclear magnitude $m_{\mathrm{v}}=22.3$ while 4.07 AU from the sun and 3.58 AU from the Earth; if we assume this to be a bare nucleus, its diameter would be $3 \mathrm{~km}$ for an albedo of $5 \%$.

Figure 5 is the cumulative plot of known close perijoves. The curve is extrapolated to Europa's orbit using Eq. (11) and an average $v_{\infty}=5 \mathrm{~km} / \mathrm{s}$. The four close approaches in the last 120 years imply 8 Io-crossers and 14 Europa crossers in the same interval. We have seen enough JFCs encounter Jupiter to say that one passes inside Europa's orbit no less often than once per decade (and that Jupiter should be getting hit about once every 100 years). The probability that a Europa-crossing JFC will actually hit Europa is $6.4 \times 10^{-6}$. Although this is the value we obtain from our Monte Carlo simulation, it is very close to what one would obtain $\left(5.5 \times 10^{-6}\right)$ by simply comparing the surface area of Europa to the area of the sphere enclosing Europa's orbit (Europa's gravitational focusing is small and Jupiter's cancels out). It follows that Europa is currently being hit by observable JFCs at a rate of about 6 per 10 million years.

By coincidence, this happens to be the same rate we obtained by other means (see Table I). This is encouraging. One might be tempted to argue that SL9 should not be counted (although one could also argue that every large 1994 fragment should be counted) - still, overall, one does not shake off the impression that 6 hits in $10 \mathrm{Ma}$ is more likely an underestimate than an overestimate. Completeness is the issue. Have Earth-bound observers discovered all the comets to approach within $2 R_{\mathrm{J}}$ of Jupiter's cloudtops in the past 120 years? Would P/Brooks 2 have been discovered had Jupiter deflected it outward rather than inward? On the other hand, the general impression one gets is that tidal disruption makes a comet briefly bright. If so, our sample may not be badly misleading.

\subsection{Uncertainties in the Cratering Rate}

The several different calibrations to the JFC impact rates discussed above are summarized in Table II. The largest uncertainty is in the masses of the comets. We have estimated masses three independent ways:

(1) If we use active magnitudes to set masses (e.g., Eqs. (19) and (20)), the uncertainty in $m$ is at least a factor of 10. This corresponds to an uncertainty in the cratering rate $\dot{C}(>D) \propto m^{-0.65}$ of at least a factor of 5 . From counting statistics and possible incompleteness, the uncertainty in the observed number of JFCs with $q<2$ and $H_{\mathrm{T}}<9$ would be about $50 \%$. Extrapolating comet numbers from $2-\mathrm{km}$ objects to the $1-\mathrm{km}$ objects that make $20-\mathrm{km}$ craters introduces an uncertainty in the cratering rate $\dot{C}(>D) \propto$ 
TABLE II

Impact Rates by 1-km JFCs on Earth and Jupiter $\left[\mathrm{yr}^{-1}\right]$

\begin{tabular}{lrc}
\hline \multicolumn{1}{c}{ Calibration } & \multicolumn{1}{c}{ Earth } & Jupiter \\
\hline SWS94 & $7 \times 10^{-7}$ & \\
$B(1,0)$ & $6.2 \times 10^{-7}$ & 0.020 \\
$H_{10}{ }^{a}$ & $4 \times 10^{-7}$ & 0.012 \\
$H_{10}{ }^{b}$ & $1 \times 10^{-7}$ & 0.003 \\
Big comets $^{c}$ & $4 \times 10^{-7}$ & 0.012 \\
Famous Perijoves $_{\text {LD97 }}$ & $3 \times 10^{-7}$ & $>0.01$ \\
This study & $3.5 \times 10^{-7}$ & 0.01 \\
& & 0.011 \\
\hline
\end{tabular}

\footnotetext{
${ }^{a}$ Eq. (19)

${ }^{b}$ Eq. (20)

${ }^{c}$ Eq. (22).
}

$D^{-2.2 \pm 0.6} \propto d^{-1.97 \pm 0.5}$, of about a factor 1.4. Together, these correspond to an uncertainty in the cratering rate of at least a factor of 6 .

(2) If we follow SW82, we estimate that the uncertainty in $m$ is at least a factor of 3 and could easily be a factor of 8.1. The uncertainty in the number of nearby active comets brighter than $B(1,0)=16$ is small, no more than $20 \%$, while as above the extrapolation from 2.2 to $1 \mathrm{~km}$ introduces an uncertainty of 1.5. The net uncertainty in the cratering rate is about a factor of 4 .

(3) If we follow KD96, the uncertainty in $m$ for the largest JFCs is smaller, about a factor of 3, but the uncertainty in the number of active comets larger than $10 \mathrm{~km}$ is at least a factor of 2 , and extrapolating comet numbers from 10- to 1-km bodies introduces another factor 3 uncertainty. Together, these correspond to an uncertainty in the cratering rate of a factor of 4 .

The second source of uncertainty, common to all three mass calibrations, is using LD97 to scale from $N(q<2)$ to impacts on Jupiter. This probably introduces an uncertainty of a factor of 2 , the chief cause of concern being the ratio of active to extinct comets. An independent measure of the comet flux at Jupiter, based on the three comets known to have closely encountered Jupiter, gives essentially the same result. The uncertainty in the ratio of impacts on a satellite to those on Jupiter we estimate to be less than $50 \%$.

The last of the major uncertainties is in the cratering efficiency. We have for complex craters $D \propto(\mathrm{m} / \rho)^{0.294}$ with an uncertainty that could be as high as $50 \%$ in $D$, which corresponds to an uncertainty in $\dot{C}(>D) \propto D^{-2.2}$ of a factor of 2.4. There are additional small uncertainties in the target density and in the way crater diameters scale with incidence angle that we will ignore in this discussion.

The combined uncertainty, $\sigma=\sqrt{\sum \sigma_{\mathrm{i}}^{2}}$, is about a factor of five. This is higher than one would like. It is not easily repaired by a single breakthrough, as the uncertainty has many causes. For comparison, SW82 derived rates that are about 30\% of what we estimate, while Shoemaker (1996; hereafter S96) derived cratering rates by JFCs that are about $30 \%$ higher than what we deduce. The ratio of JFC cratering rates in S96 to those in SW82 is a factor of 4.5 (the difference due to a higher estimate of the number of extinct comets).

It is interesting and perhaps surprising that three fundamentally independent measures of comet size-SW82's calibration of $B(1,0)$, the various active magnitude-mass relations, and the directly measured nuclear sizes of the largest active JFCs-should agree so well. All three approaches give $\dot{N}_{\mathrm{J}}(d>1) \approx 0.01 \mathrm{yr}^{-1}$; i.e., the impact rate of km-size comets on Jupiter is about one per century. Because the three separate means of measuring $m$ agree, the true uncertainty in the cratering rate may be smaller than we have quoted (but it could also mean that everyone is wrong).

\section{NEARLY ISOTROPIC COMETS}

The nearly isotropic comets (to be distinguished from the ecliptic comets, Levison 1996) come from the Oort cloud, a spherically symmetric population that surrounds the Sun at a distance of some 40,000 AU. A long period comet (LPC) is traditionally defined as a comet with a period longer than 200 years. Halley-type comets (HTCs) are comets with $T<2$ and periods shorter than 200 years. LPC orbits are nearly parabolic and more or less isotropically inclined. The inclinations of the HTCs are less random, showing a slight preference for prograde orbits.

\subsection{Long Period Comets}

The average LPC makes about five passages through the inner solar system ( $q<4$ AU; Weissman 1991); although most are scattered or disintegrated, some evolve inward more or less indefinitely. Dynamical studies of LPC orbital evolution imply that first-time visitors are overrepresented among LPCs as a whole (e.g., Weissman 1991, Wiegert and Tremaine 1997). According to Wiegert and Tremaine (1997), the mean number of passes inside of 3 AU by an indestructable LPC would be 40 . This "fading problem" dates to Oort's original work, and it is not at present resolved. To some extent how one treats the fading problem affects our estimated impact rates. If we take the ratio of expected (40) to observed (5) returns, we could have as many as 8 inactive LPCs for each active one.

Impact probabilities and impact velocities. Our approach to generating relative impact probabilites and impact velocities for LPCs parallels our approach for JFCs, but there are two steps. The first step is heliocentric: we generate random orbits assuming isotropic parabolic or- 
bits. Relative impact probabilities and absolute encounter velocities with the planets are computed using Öpik's formulae for parabolic orbits (Eqs. (2-5) with $e=1$ ). We use two different perihelion distributions. The expected perihelion distribution of new parabolic comets is uniform in $q$. But most LPCs are not making their first perihelion passage. Observationally inspired descriptions include $N(<q) \propto q^{1.5}$ (Kresak and Pittich 1978) and $N(<q) \propto$ $500 q-175 ; q>1$ (SW82). According to these latter, the ratio of LPCs crossing Jupiter's orbit to those crossing Earth's is 11.5 and 8, respectively. These three estimates imply a factor two uncertainty in the relative impact rate on Jupiter vs Earth. We will use the nonuniform $N(<q)$ $\propto q^{1.5}$ distribution as an upper bound, and the uniform distribution as a lower bound.

In the second step, relative impact probabilities and absolute impact velocities with the Galilean satellites are computed exactly as was done for JFCs, save that we use the encounter velocity generated in the first step. This makes the implicit assumption that the apex-antapex asymmetry in the LPC encounter velocities with Jupiter average out over a satellite's orbit. This issue would be of more concern if LPCs were important to cratering the Galilean satellites.

The impact velocity distribution that results is shown in Fig. 6. Impact probabilities and other results for both the uniform and nonuniform perihelion distributions are listed in Table III.

Mass and number. SW82 estimate that the number of LPCs passing within $1 \mathrm{AU}$ annually is

$$
\dot{N}(q<1,>d)=30(d / 2.5 \mathrm{~km})^{-1.97}
$$

and the number of LPCs crossing Jupiter's orbit is

$$
\dot{N}(q<5.5,>d)=230(d / 2.5 \mathrm{~km})^{-1.97} .
$$

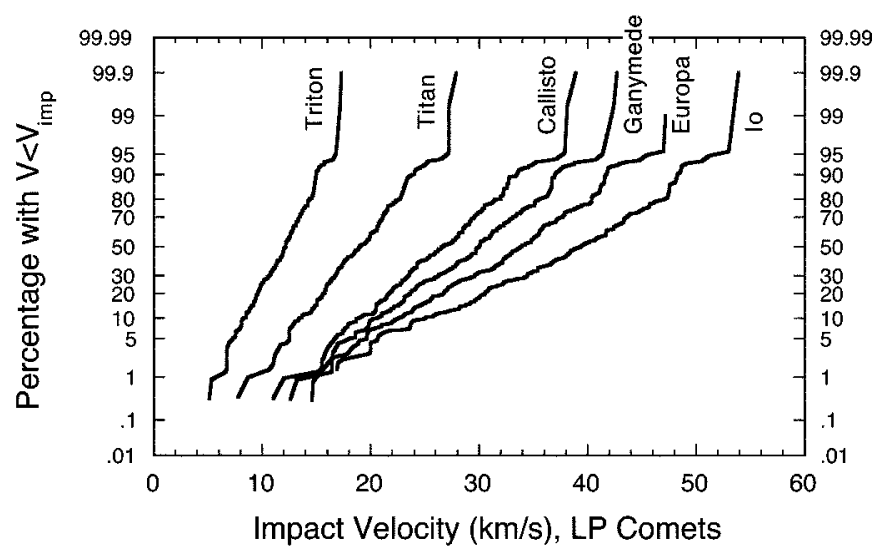

FIG. 6. Cumulative impact velocity distributions for impacts by long period or Halley-type comets on various satellites in the solar system.
These estimates are based on Roemer's $B(1,0)$ magnitudes and have been corrected for coma by reducing the number of objects by a factor of 8.1. In this we have followed SW82 precisely. The correction is the same as SW82 used for JFCs. We also quote the same power law for the mass distribution that they do. SW82 do not explicitly consider extinct LPCs.

The average probability that Earth will hit a new Oort cloud comet is $2.3 \times 10^{-9}$ per apparition $(q<1)$; for the catalog of observed LPCs, the impact probability is somewhat higher at $3 \times 10^{-9}$ per apparition (Olsson-Steel 1987, Steel 1993). SWS94 repeat this estimate. They also cite IRAS-Araki-Alcock, a weakly active 9-km diameter comet that passed only 0.031 AU from Earth, as supporting evidence of a high LPC flux near Earth. According to Eq. (23), a 0.031 AU approach by a 9-km diameter comet would be a 300 -year event.

On the other hand, more recent anecdotal evidence argues that the rates given in Eqs. (23) and (24) are high. Hyakutake, a 2- to 3-km object passing only about $0.1 \mathrm{AU}$ from Earth, would be a 2-year event. Hale-Bopp, usually described as a $\sim 40 \mathrm{~km}$ body (Weaver et al. 1997), and which passed perihelion inside $1 \mathrm{AU}$, would be an 8 year event according to Eq. (23). This certainly seems wrong-is it really possible that inactive $40-\mathrm{km}$ comets are slipping past our view every few years? The observed frequency of Hale-Bopps at 1 AU appears to be more like 100 years. The Great Comet of 1811 was similar to Hale-Bopp, and two naked eye 18th-century comets with perihelia at 2.2 AU (1747) and 4.05 AU (1729) may have been larger (Kronk 1984, Kidger 1997). In all likelihood SWS94's LPC fluxes are too high by as much as an order of magnitude.

Bailey et al. (1994) gave alternative calibrations based on active absolute magnitudes $H_{10}$, but their numbers do not appear to be internally self-consistent; our best guess is that Bailey et al.'s preferred distribution is equivalent to

$$
\dot{N}(q<1,>d)=2.5(d / 2.5 \mathrm{~km})^{-1.9} .
$$

This makes the passage of a $100-\mathrm{km}$ diameter comet inside $1 \mathrm{AU}$ a 400-year event, as they claim it is. According to Eq. (25) Hale-Bopp is an 80-year event, Hyakutake a 20year event, and IRAS-Araki-Alcock a 3000-year event.

The latter is so infrequent as to be a little worrisome, but it should be pointed out that IRAS-Araki-Alcock was a relatively inactive comet in a relatively strongly bound orbit ( $a=95 \mathrm{AU})$, dynamically more akin to an HTC than to a new LPC. SWS94, at least, suggested that extinct comets are as numerous among the HTCs as they are among the JFCs. IRAS-Araki-Alcock appears to be on the way toward becoming an example; the recently discovered 1996PW (an asteroid with $a=327$ AU) may be an example of the real thing (Weissman and Levison 1996).

There is a third choice in the literature, one that is even 
TABLE III

Long Period Comets

\begin{tabular}{|c|c|c|c|c|c|c|}
\hline & Earth & Jupiter & Io & Europa & Ganymede & Callisto \\
\hline$\left\langle v_{\mathrm{i}}\right\rangle^{a}$ & 55 & 64 & 39 & 34 & 30 & 27 \\
\hline$m_{\mathrm{i}}(D=20)^{b}$ & $2.4 \times 10^{15}$ & & $1.8 \times 10^{15}$ & $2.6 \times 10^{14}$ & $3.5 \times 10^{14}$ & $3.8 \times 10^{14}$ \\
\hline$d_{\mathrm{i}}(D=20)^{c}$ & 1.8 & & 1.64 & 0.86 & 0.94 & 0.96 \\
\hline \multicolumn{7}{|c|}{ Uniform perihelion distribution, $N(>q) \propto q$} \\
\hline$P_{\mathrm{i}}^{d}$ & $2.3 \times 10^{-9}$ & $1.0 \times 10^{-7}$ & $1.9 \times 10^{-11}$ & $1.1 \times 10^{-11}$ & $2.4 \times 10^{-11}$ & $1.8 \times 10^{-11}$ \\
\hline$P_{\mathrm{i}}(d>1)^{e}$ & $6.0 \times 10^{-7}$ & $1.4 \times 10^{-4}$ & $2.6 \times 10^{-8}$ & $1.5 \times 10^{-8}$ & $3.4 \times 10^{-8}$ & $2.5 \times 10^{-8}$ \\
\hline$\tau(D>20)^{f}$ & $6 / 60$ & & $120 / 1200$ & $50 / 500$ & $25 / 250$ & $37 / 370$ \\
\hline$\dot{C}(D>10)^{g}$ & $1.5 \times 10^{-15}$ & & $9.7 \times 10^{-16}$ & $3.1 \times 10^{-15}$ & $2.0 \times 10^{-15}$ & $1.7 \times 10^{-15}$ \\
\hline$h$ & $1.5 \times 10^{-16}$ & & $9.7 \times 10^{-17}$ & $3.1 \times 10^{-16}$ & $2.0 \times 10^{-16}$ & $1.7 \times 10^{-16}$ \\
\hline \multicolumn{7}{|c|}{ Nonuniform perihelion distribution, $N(>q) \propto q^{1.5}$} \\
\hline$P_{\mathrm{i}}^{d}$ & $2.7 \times 10^{-9}$ & $1.2 \times 10^{-7}$ & $2.3 \times 10^{-11}$ & $1.3 \times 10^{-11}$ & $3.0 \times 10^{-11}$ & $2.2 \times 10^{-11}$ \\
\hline$P_{\mathrm{i}}(d>1)^{e}$ & $6.0 \times 10^{-7}$ & $3.2 \times 10^{-4}$ & $6.2 \times 10^{-8}$ & $3.6 \times 10^{-8}$ & $7.9 \times 10^{-8}$ & $5.8 \times 10^{-8}$ \\
\hline$\tau(D>20)^{f}$ & $6 / 60$ & & $50 / 500$ & $20 / 200$ & $10 / 100$ & $16 / 160$ \\
\hline$\dot{C}(D>10)^{g}$ & $1.5 \times 10^{-15}$ & & $2.3 \times 10^{-15}$ & $7.4 \times 10^{-15}$ & $4.8 \times 10^{-15}$ & $4.0 \times 10^{-15}$ \\
\hline$h$ & $1.5 \times 10^{-16}$ & & $2.3 \times 10^{-16}$ & $7.4 \times 10^{-16}$ & $4.8 \times 10^{-16}$ & $4.0 \times 10^{-16}$ \\
\hline
\end{tabular}

${ }^{a}$ Average impact velocity $[\mathrm{km} / \mathrm{s}]$.

${ }^{b}$ Impactor mass $[\mathrm{g}]$ giving $D=20 \mathrm{~km}$ crater at $\theta=45^{\circ}$.

${ }^{c}$ Impactor diameter $[\mathrm{km}]$ at $\rho_{\mathrm{i}}=0.8 \mathrm{~g} / \mathrm{cm}^{3}$.

${ }^{d}$ Impact probability per perihelion passage.

${ }^{e}$ Impact probability $d>1 \mathrm{~km}\left[\mathrm{yr}^{-1}\right]$.

${ }^{f}$ Timescale [Ma] for $D>20 \mathrm{~km}$ craters [high/low flux].

${ }^{g}$ High cratering rate, $D>10 \mathrm{~km}\left[\mathrm{~km}^{-2} \mathrm{yr}^{-1}\right]$, based on Eq. (23).

${ }^{h}$ Low cratering rate, $D>10 \mathrm{~km}\left[\mathrm{~km}^{-2} \mathrm{yr}^{-1}\right]$, based on Eq. (25).

more inextricably linked to the mass-magnitude relation Eq. (19). Weissman (1990) estimated that 10 LPCs brighter than $H_{10}=11$ cross Earth's orbit each year. Weissman favored a distribution in which very bright comets are relatively rare (Everhart 1967). The knee in the distribution is at $H_{10}=5.4$. If we simply go by absolute magnitude, Hale-Bopp, with $H=-1.3$ (Kidger 1997), would be a 20,000 year event (and a $100 \mathrm{~km}$ diameter comet, according to Eq. 19). Weissman's distribution looks better if we ignore Hale-Bopp's magnitude: For $\rho=0.6$ (the density Weissman uses), the frequency of passes within $1 \mathrm{AU}$ are

$$
\begin{array}{ll}
\dot{N}(q<1, \geq d)=10(d / 2.33 \mathrm{~km})^{-1.95} & d<13 \mathrm{~km} \\
\dot{N}(q<1, \geq d)=0.35(d / 13 \mathrm{~km})^{-4.3} & d>13 \mathrm{~km}
\end{array}
$$

For small comets these rates are the geometric mean between Shoemaker's and Bailey et al.'s. In this distribution Hale-Bopp is a 350-year event, Hyakutake a 6-year event, and IRAS-Araki-Alcock a 1000-year event.

The impact probability of LPCs and Jupiter is $1.0 \times$ $10^{-7}$ per comet per apparition for a uniform perihelion distribution and $1.2 \times 10^{-7}$ for Kresak and Pittich $N(>q) \propto q^{1.5}$ (see Table III). According to Eq. (24), the higher estimate, for uniform perihelia the impact rate on Jupiter should be

$$
\dot{N}_{\mathrm{J}}(>d)=1.4 \times 10^{-4}(d / 1 \mathrm{~km})^{-1.97}
$$

This is $1.5 \%$ of the impact rate by JFCs. For the $N(>q) \propto$ $q^{1.5}$ distribution, the rate is somewhat higher,

$$
\dot{N}_{\mathrm{J}}(>d)=3.2 \times 10^{-4}(d / 1 \mathrm{~km})^{-1.97} .
$$

Impacts by LPCs are relatively more important on satellites than on Jupiter.

Shoemaker's high LPC flux provides a useful upper limit. It can be taken straight, or it can be regarded as implicitly including inactive LPCs. According to Eqs. (27) and (28), about $5-10 \%$ of the craters on Callisto would be made by LPCs; fewer for the other satellites. These rates are reduced by an order of magnitude if we follow Eq. (25) for the number of LPCs. The latter is probably a lower limit, as it omits inactive comets. Cratering timescales for both the high and low estimates are quoted in Table III. In all likelihood LPCs make their presence felt in the jovian system at the $1-10 \%$ level.

\subsection{Halley-type Comets}

Halley-type comets are important for cratering Earth. There are only about two dozen known, most of which are both Earth-crossing and bright. The apparent lack of faint 
HTCs, as compared to the JFCs and LPCs, implies a real absence of old small HTCs; to the extent that kilometersize bodies are missing, the estimated production rate of 10 - to $20-\mathrm{km}$ craters is affected.

By origin HTCs appear to be the short period tail of the LPCs. In particular, a Kuiper Belt source does not produce a good match to the HTC orbital distribution (LD97). As a class HTCs retain high inclinations, although the inclination distribution deviates considerably from isotropy. What is important to us here is that, as a class, they do not appear to cluster around Jupiter, as the JFC's do. Rather, their perihelion distribution is indistinguishable from that of LPCs.

The 15 Earth-crossing HTCs do so once every 2-4 years, with about half of these passes by $\mathrm{P} /$ Machholz 1 , a short period comet which with $T=1.94$ is probably an HTC. But $\mathrm{P} / \mathrm{Machholz} 1$ is faint $(H=13)$. The rate at which bright $H<10.5$ HTCs cross Earth's orbit is about one every six years, while the corresponding rate for LPCs is three per year (Fernandez and Ip 1991). Hence the ratio of bright LPCs to HTCs is about 20. SWS94 argue that the fraction of HTCs that are extinct should be about the same as for JFCs. At least two inactive HTCs are known, and Hidalgo itself, at $T=2.07$, could qualify as either JFC or HTC. If we take the ratio of extinct to active HTCs to be 20 , the net result is that the impact rate by large HTCs is about the same as the impact rate by active LPCs. For the present we will assume that the impact rate by HTCs is equal to our lower estimate for impacts by LPCs. But, as noted above, most known HTCs are bright. The importance of kilometer-size HTCs may be much smaller.

\section{ASTEROIDS}

The trojan asteroids orbit the sun in two clusters, one leading and the other trailing Jupiter by $60^{\circ}$. Trojans can stray, either by dynamical chance or by intra-trojan collisions (Marzari et al. 1995). After they escape they follow orbits like those of the Jupiter family comets $(T \approx 3)$. Thus the trojans can be regarded as an alternative source for JFCs (Rabe 1972, Marzari et al. 1995). Escaped trojans were simulated by Levison et al. (1997; hereafter LSS97). LSS97 assumed that trojans follow the same $N(>d) \propto$ $d^{-1.97}$ size distribution that Shoemaker assumes for JFCs. They stated their results in two ways: (i) about 1 JFC in 150 is an escaped trojan, and (ii) there are now about a dozen kilometer-size trojans in orbits with $q<2.5 \mathrm{AU}$; i.e., $N(q<2.5, d<1)=12$. The former indicates that trojans are not likely to be important. But we will start with the second in order to be internally consistent.

LD97 find that, for active JFCs, $N(q<2.5) / N(q<2)=$ $11 / 4$; they also find that for $q<2.5$ the ratio of extinct to active JFCs is 3.5. The ratio of trojans to all JFCs (extinct and active $)$ would therefore be $12 /(110 \times 4.5)=1 / 40$.
Hence if trojans follow the cometary $N(>d) \propto d^{-1.97}$ size distribution, they make up 2-3\% of the impacts on Jupiter.

The above may be an underestimate. If trojans are a collisionally evolved population, they would be expected to obey the $N(>d) \propto d^{-2.5}$ distribution that theory imposes on a fragmentation cascade (Dohnanyi 1972, Safronov 1972, Williams and Wetherill 1994, Tanaka et al. 1996, Durda and Dermott 1997). The observed trojans are large bodies, of order $100 \mathrm{~km}$. If the $N(>d) \propto d^{-2.5}$ holds from 1 to $100 \mathrm{~km}$, the number of kilometer-size trojans would exceed LSS97's estimate by a factor 10. There would be 120 kilometer-sized trojans passing within 2.5 AU, and trojans would be $25 \%$ as important as JFCs for $20 \mathrm{~km}$ craters at Jupiter. If very small JFCs prove to be rare, trojans may be the main source of small primary craters on the Galilean satellites.

Main belt asteroids. Stray asteroids from the main belt are difficult to assess. The dynamical models are not yet very successful at reproducing the observed population of NEAs (Gladman et al. 1997). The models are useful nevertheless. In particular, they indicate that main belt asteroids are not especially efficient at hitting Jupiter. Individually, asteroids are no more likely to hit Jupiter than any of the other objects discussed here. As a consequence, the relatively small mass of the main belt imposes a strong upper limit to cratering of the Galilean satellites by asteroids.

Gladman et al. (1997) studied the evolution of asteroid orbits that originate from unstable resonances that slice through the identified collisional families of asteroids. Their original purpose was to identify the sources of the NEAs, but this proved an unreachable goal. What they found instead is that lifetimes of asteroids in strong resonances are very short. Each resonance is different, but to first order those nearer the Sun tend to produce asteroids that hit the Sun while those farther from the Sun tend to send asteroids to the outer Solar System or beyond. A few of the inbound objects hit terrestrial planets. A few of the outbound objects hit Jupiter. The division between domains is around 2.6 AU, but most resonances will send some objects in either direction.

Gladman et al. (1997) found that about 1-2\% of the outbound asteroids hit Jupiter. This fraction of hits is consistent with the results obtained by LD97 for JFCs. As the orbits of asteroids encountering Jupiter are likely to be essentially similar to the orbits of JFCs encountering Jupiter, this is an encouraging result. As shown above, the impact rate on Ganymede is about $10^{-4}$ that on Jupiter. Therefore it takes about a million kilometer-size outbound asteroids to create a $20-\mathrm{km}$ crater on Ganymede. ${ }^{2}$ This

\footnotetext{
${ }^{2}$ To be more precise, what is required are $4 \times 10^{14} \mathrm{~g}$ impactors, which implies a $750 \mathrm{~m}$ diameter asteroid at $\rho=1.7 \mathrm{~g} / \mathrm{cm}^{3}$.
} 
ratio of one in a million is a nice reminder that planetary accretion in the contemporary solar system is inefficient. To produce a million kilometer-size asteroids requires the disruption of a parent body with mass of order $10^{22} \mathrm{~g}$, about $200-\mathrm{km}$ in diameter. In other words, it takes the disruption of a $200-\mathrm{km}$ diameter asteroid in a resonance to produce a single $20-\mathrm{km}$ crater on Ganymede. It is difficult to plausibly produce hundreds or thousands of 20$\mathrm{km}$ craters this way. To put this in perspective, there are currently only $\sim 30$ asteroids larger than $200 \mathrm{~km}$ (Cellino et al. 1991).

In order to quantify the asteroid impact rate on Ganymede, we can attempt to scale from the NEA impact rate on Earth. This is a highly speculative exercise, because we do not know the source or sources of the NEAs, and the resonances that supply the NEAs are probably not the same resonances that divert asteroids to Jupiter. Jupiter gets hit by asteroids about 0.1 to $>100$ times as often as Earth, depending on the resonance (Gladman et al. 1997). As a generous rough estimate we will assume that the net factor is 10 , so that Ganymede gets struck by an asteroid about $10^{-3}$ as often as Earth. A recent estimate of the terrestrial cratering rate in the past $500 \mathrm{Ma}(D>20 \mathrm{~km})$ is $4.5 \pm 2 \times 10^{-15} \mathrm{~km}^{-2} \mathrm{yr}^{-1}$ (Grieve and Shoemaker 1994). A $20-\mathrm{km}$ crater on Earth scales to a $50-\mathrm{km}$ crater on Ganymede. So the cratering rate $(10-\mathrm{km}$ craters $)$ on Ganymede is $4.5 \times 10^{-15} \times 10^{-3} \times(50 / 10)^{2.2}=1.6 \times 10^{-16}$ $\mathrm{km}^{-2} \mathrm{yr}^{-1}$. This is to be compared to the comparable JFC rate in Table I of $7 \times 10^{-14} \mathrm{~km}^{-2} \mathrm{yr}^{-1}$. As expected, the contribution of main belt asteroids is negligible.
Obviously things may have been different in the early solar system. It is usually presumed that the current low mass of the asteroid belt does not reflect its original mass. An augmented ancient asteroid belt makes a possible source for Earth's volatiles (Wetherill 1994, Zahnle 1998) and asteroid showers are one (of many) possible candidates for the late lunar heavy bombardment. An ancestral outer asteroid belt comparable to the mass of the Earth seems plausible. At a part in a million, Ganymede would recruit $10^{22} \mathrm{~g}$ of this material, enough to produce a million $20-\mathrm{km}$ craters. But it is difficult to write usefully about hypothetical events occurring at $4.5 \mathrm{Ga}$. Certainly other sources of stray bodies would also have been more important long ago, some of which are now extinct, and it is very hard to assess how important the vanished outer asteroid belt may have been, or when.

\section{SUMMARY OF IMPACT RATES}

Our impact rates for the various sources are listed in Table IV; Table $\mathrm{V}$ gives a more complete breakdown for Europa. The rates are given in terms of $10-\mathrm{km}$ craters, assuming the crater counts follow the $N(>D) \propto D^{-2.2}$ power law. This diameter and power law are used to ensure consistency with Shoemaker's work.

Low estimates are given for both LPCs and HTCs. But even if the high LPC impact rates were adopted, LPCs would make only a minor contribution to cratering. Trojans could be more important, if small trojans follow the canonical fragment mass distribution and small comets do not.

TABLE IV

Cratering Rates $\left(D>10 \mathrm{~km}\left[\mathrm{~km}^{-2} \mathrm{yr}^{-1}\right]\right)$

\begin{tabular}{lcccr}
\hline & Io & Europa & Ganymede & \multicolumn{1}{c}{ Callisto } \\
\hline JFCs $^{a}$ & $4.5 \times 10^{-14}$ & $1.0 \times 10^{-13}$ & $5.4 \times 10^{-14}$ & $2.5 \times 10^{-14}$ \\
LPCs $^{b}$ & $7.0 \times 10^{-16}$ & $2.4 \times 10^{-15}$ & $1.5 \times 10^{-15}$ & $1.3 \times 10^{-15}$ \\
HTCs $^{c}$ & $2.3 \times 10^{-16}$ & $7.4 \times 10^{-16}$ & $4.8 \times 10^{-16}$ & $4.0 \times 10^{-16}$ \\
Asteroids $^{d}$ & $1.1 \times 10^{-16}$ & $3.0 \times 10^{-16}$ & $1.6 \times 10^{-16}$ & $7.4 \times 10^{-17}$ \\
Trojans $^{e}$ & $1.4 \times 10^{-15}$ & $3.0 \times 10^{-15}$ & $1.6 \times 10^{-15}$ & $7.5 \times 10^{-16}$ \\
Total & $4.8 \times 10^{-14}$ & $1.1 \times 10^{-13}$ & $6.0 \times 10^{-14}$ & $2.7 \times 10^{-14}$ \\
& & & & \\
SW82 $^{f}$ & $1.6 \times 10^{-14}$ & Previous Estimates & & $0.3 \times 10^{-14}$ \\
SW82 $^{a}$ & $5.2 \times 10^{-14}$ & $1.3 \times 10^{-14}$ & $0.6 \times 10^{-14}$ & $1.2 \times 10^{-14}$ \\
NY95 $^{f}$ & $5.0 \times 10^{-15}$ & $4.5 \times 10^{-14}$ & $2.3 \times 10^{-14}$ & $3.1 \times 10^{-15}$ \\
NY95 $^{a}$ & $1.8 / 8 \times 10^{-14}$ & $9.0 \times 10^{-15}$ & $6.0 \times 10^{-15}$ & $1 / 4.5 \times 10^{-14}$ \\
S96 $^{-14}$ & $0.3 / 1.3 \times 10^{-13}$ & $2 / 9 \times 10^{-14}$ & $4.9 \times 10^{-14}$ \\
\hline
\end{tabular}

${ }^{a}$ Active plus extinct comets.

${ }^{b}$ Nonuniform perihelion distribution and geometric mean of high and low LPC flux.

${ }^{c}$ Assumed same as low LPC flux.

${ }^{d}$ As scaled from NEAs.

${ }^{e}$ Assumed as $3 \%$ of JFCs.

${ }^{f}$ Active comets only. 
TABLE V

Cratering Rates on Europa $\left(D>10 \mathrm{~km}\left[\mathrm{~km}^{-2} \mathrm{yr}^{-1}\right]\right)$

\begin{tabular}{lccr}
\hline & SW82 & S96 & This work \\
\hline Active JFCs & $1.3 \times 10^{-14}$ & $1.3 \times 10^{-14}$ & \\
Extinct JFCs & $2.5 \times 10^{-14}$ & $1.6 \times 10^{-13}$ & \\
Total JFCs & $3.8 \times 10^{-14}$ & $1.7 \times 10^{-13}$ & $1.0 \times 10^{-13}$ \\
LPCs & $7.0 \times 10^{-15}$ & $7.0 \times 10^{-15}$ & $3.0 \times 10^{-15}$ \\
Active HTC & & $1.0 \times 10^{-15}$ & \\
Extinct HTCs & & $1.1 \times 10^{-14}$ & \\
Total HTCs & & $1.2 \times 10^{-14}$ & $1.0 \times 10^{-15}$ \\
Asteroids & & & $<1.0 \times 10^{-15}$ \\
Trojans & & & $3.0 \times 10^{-15}$ \\
Total & $4.5 \times 10^{-14}$ & $2.0 \times 10^{-13}$ & $1.1 \times 10^{-13}$ \\
\hline
\end{tabular}

We have estimated the impact rate of trojans to be 2.5-25\% that of JFCs. The rate listed in the table is in between.

For comparison, other cratering rates were culled from the literature. The rates from SW82 are precisely as given in their Table 10.7. The other entries require some explanation. Shoemaker (1996) gave only the rates for Europa. The key difference between the newer analysis and SW82 is that the more recent analysis includes a much greater contribution from extinct JF comets. In SW82 impacts by extinct comets were 1.9 times as frequent as those by active comets; in the more recent work this ratio jumps to 14 . We have used the new ratio to prepare table entries for Io, Callisto, and Ganymede.

NY95 gave cratering rates for Amalthea, Ganymede, and Callisto. They use a crater scaling relation that is essentially identical to the relation we use here for transient craters, Eq. (12), if the leading constant is taken to be 1.8. They do not account for complex craters. NY95 obtained comet masses using one of the older versions of the massmagnitude relation,

$$
\log _{10}(m)=19.0-0.4 H_{10}
$$

this one gives similar masses for $1-\mathrm{km}$ comets to those obtained using Eq. (20).

NY95 also gave relative impact rates for all the satellites. From these we have estimated the corresponding cratering rates for Europa and Io. A peculiarity of NY95's estimates is that they neglect the existence of extinct or inactive comets. In order to compare their estimates to ours and those of Shoemaker, we have presented a separate line in the table in which their cratering rates for active comets are multiplied by factors of 3.5 and 15, to account for the relative contribution of extinct comets according to LD97 and SWS94, respectively. Even with extinct comets taken into account, NY95's cratering rates are lower than ours, in part because Eq. (29) gives relatively small comets.

\section{APEX-ANTAPEX ASYMMETRY}

One expects that the cratering rate should be higher on the leading hemisphere of a synchronously rotating satellite than on the trailing hemisphere, because the moons are revolving through an isotropic swarm of comets. The expected apex-antapex asymmetry is not a subtle effect. By contrast, for an approximately coorbiting swarm, cratering asymmetries are second order (Horedt and Neukum 1984).

In Jupiter's rest frame, the satellite has velocity $v_{\text {orb }}$ in the direction of the apex of motion, while the velocity distribution of the comets at $a_{\text {sat }}$ is a sphere of radius $v_{\mathrm{c}}=\sqrt{2 v_{\text {orb }}^{2}+v_{\infty}^{2}}$. The normal component of the impact velocity at a point on the satellite's surface is

$$
v_{\perp}=v_{\text {orb }} \cos \beta-v_{\mathrm{c}} \cos \theta,
$$

where $\beta$ is the angular distance on the surface measured from the apex, and $\theta$ is the angle on the comet velocity sphere measured from the surface normal. Impacts occur when $v_{\perp}>0$. The instantaneous flux of bodies is proportional to $v_{\perp}$.

For large craters, crater diameter goes as $v_{\perp}^{0.5}$ (Eq 14). We seek the cratering rate at a given diameter. If the cumulative number of craters goes as $N(>D) \propto D^{-\gamma}$ (we have been following Shoemaker and using $\gamma=2.2$ ), the cratering rate at a given diameter $D_{\text {。 goes as }} \dot{C}\left(>D_{\circ}\right) \propto$ $v_{\perp}^{0.5 \gamma} \approx v_{\perp}^{1.1}$. The cratering rate at $\beta$ should then be proportional to the average value of $v_{\perp}^{1+0.5 \gamma}$ integrated over the accessible part of the velocity sphere,

$$
\left\langle v_{\perp}^{1+0.5 \gamma}(\beta)\right\rangle=\frac{\int_{0}^{\theta^{\prime}} \sin \theta d \theta\left(v_{\text {orb }} \cos \beta-v_{\mathrm{c}} \cos \theta\right)^{1+0.5 \gamma}}{\int_{0}^{\theta^{\prime}} \sin \theta d \theta} .
$$

The resulting cratering rate as a function of $\beta$ is

$$
\dot{C}(\beta)=\dot{C}(\pi / 2)\left(1+\frac{v_{\text {orb }}}{v_{\mathrm{c}}} \cos \beta\right)^{1+0.5 \gamma} .
$$

For the JFCs, for which $v_{\infty}^{2} \ll v_{\text {orb }}^{2}$ and hence $v_{\mathrm{c}} \approx$ $\sqrt{2} v_{\text {orb }}$, the ratio of the cratering rate at the apex to the cratering rate at the antapex is approximately

$$
\frac{\dot{C}(0)}{\dot{C}(\pi)} \approx\left(\frac{\sqrt{2}+1}{\sqrt{2}-1}\right)^{2} \approx 34
$$

more precise values range from 32 for Io to 20 for Callisto. These compare to SW82's 38 for Io and 10 for Callisto. We do not know the origin of the difference, although some of the difference may be that SW82 use higher $v_{\infty}$. 
Horedt and Neukum (1984) derive smaller asymmetries, ranging from 15 to 7 for Io to Callisto; again, we do not know the origin of the difference.

Note that the apex-antapex asymmetry is itself asymmetrical. Most of the asymmetry is that craters avoid the antapex. The cratering rate at the apex is about 2.5 times the global average, but the cratering rate at the antapex is only $10 \%$ of the global average. This occurs because crater size depends on impact velocity, and antapex impact velocities are generally quite low.

Equation (32) should apply for any low-energy heliocentric population (JFCs, trojans, main belt asteroids). It should also apply approximately to LPCs if we take $v_{\infty}$ appropriately; a fair approximation is $v_{\infty} \sim 24 \mathrm{~km} / \mathrm{s}$. The resulting apex-antapex asymmetries range from 10 at Io to 3.7 at Callisto. Equation (32) does not apply to jovicentric debris, for which no strong apex-antapex asymmetry is expected (Horedt and Neukum 1984).

\section{DISCUSSION}

Io. There are no reports of impact craters on Io. Resurfacing is not instantaneous, even on Io. As the resurfacing rate can be estimated by other means (e.g., from its heat flow, observed resurfacing rate, or even (in the far future) radiometrically dated rocks), Io provides an independent constraint on the cratering rate (Johnson and Soderblom 1982). Resurfacing of Io during the past 20 years has been estimated as $1.3 \mathrm{~cm} / \mathrm{yr}$ based on Io's heat flow and the evidence that resurfacing is dominated by silicate flows (Blaney et al. 1995). This needs to be compared with our predicted cratering rate. Our nominal result is that $20-\mathrm{km}$ craters form on Io every 2.5 Ma. For comparison, on the Moon 20-km craters form every 8-12 Ma (McEwen et al. 1997); i.e., the Ionian cratering rate is about four times higher than the lunar cratering rate. At this rate, to erase all impact craters observable but not seen by Voyager, Io's resurfacing rate would need to exceed $0.4 \mathrm{~cm} / \mathrm{yr}$ (Johnson and Soderblom, 1982). For the present, there is no contradiction.

Europa. Our nominal result for Europa is that $20-\mathrm{km}$ craters form on a 1.4 myr timescale (Table I). As there appear to no more than five or ten $20-\mathrm{km}$ craters on Europa, its nominal surface age would be about $10 \mathrm{Ma}$. As discussed above, the uncertainty in this estimate is about a factor of five. Nonetheless, it would be very difficult to contrive matters such that Europa could be aged beyond $100 \mathrm{Ma}$. In sum, and this too is not a new observation, Europa's surface is clearly young.

Ganymede. With Ganymede we are dealing with surfaces where uncertainties of a factor of five make an important qualitative difference. It is sometimes implicitly or explicitly assumed that the lunar late heavy bombardment was a Solar-System-wide phenomenon, so that the details of the lunar chronology can be applied generally throughout the solar system. Ganymede is reminiscent of the Moon in its mix of ancient cratered terrains and relatively sparsely cratered terrains, and in its mix of ancient cratered basins and younger relatively sparsely cratered impact basins. In this sense the large young Gilgamesh basin is stratigraphically similar to the lunar Orientale basin, and so it has come to pass that, because the Orientale basin is $3.8 \mathrm{Ga}$, this same age gets assigned to Gilgamesh. The analogy has been pursued in explicit detail by forcing ganymedean cratering to obey the temporal evolution determined for lunar craters (Shoemaker et al. 1982, Neukum et al. 1997). But Ganymede is not the Moon, and Gilgamesh is not Orientale. For one thing, the Gilgamesh event was much smaller than the Orientale event, an order of magnitude less in energy. (Gilgamesh is smaller than Orientale and it is in a lower density material.)

To discuss Ganymede more fully, we need to account for secular changes in the impact rate through time. Our estimates for the age of Europa are based on the current flux of objects from the Kuiper Belt. LD97 find that the characteristic timescale for these objects to evolve from instability to their various fates is about $45 \mathrm{Ma}$, and so the current JFC flux should provide an adequate estimate of conditions over the past $10 \mathrm{Ma}$ at Europa. This will not suffice for Ganymede. It is certainly possible that the present state is atypical, and that present impact rates are very high (or very low). Such variability would directly affect our estimate of surface ages on Ganymede. But here, all we can do is acknowledge the possibility. According to simulations by Holman and Wisdom (1993), a collisionless Kuiper Belt dissipates as $t^{-1}$. Collisions among Kuiper Belt objects would tend to increase the dissipation rate (Stern and Colwell 1997). We will use $t^{-1}$ to extrapolate the JFC flux back in time. If the observed crater density is $C(>D)$ and the current cratering rate is $\dot{C}_{0}(>D)$, the current cratering timescale is $\tau_{\circ}=C(>D) / \dot{C}_{\circ}(>D)$. This would be the age of a surface for constant $\dot{C}(>D)$. For $\dot{C}(>D)=\dot{C}_{\mathrm{o}}(>D) \times$ $t_{\mathrm{o}} / t$, where $t_{\mathrm{o}} \approx 4.56 \mathrm{Ga}$ is the age of the solar system, the surface age $\tau$ would be

$$
\tau=t_{\circ}\left(1-\exp \left(-\tau_{\circ} / t_{\circ}\right)\right)
$$

Note that $\dot{C}(>D)$ is a function of the angular distance $\beta$ from the apex of motion.

Figure 7 plots crater densities on various types of surfaces on Ganymede and Callisto as a function of apex angle $\beta$. Data are those tabulated by Shoemaker et al. (1982). All data are expressed in terms of the equivalent number of $10-\mathrm{km}$ craters expected for Shoemaker's $N(>D) \propto D^{-2.2}$ power law. The figure also shows the surface ages that correspond to these crater densities as a function of $\beta$, calculated according to Eq. (32), and making 
Surface Ages of Europa, Ganymede, and Callisto

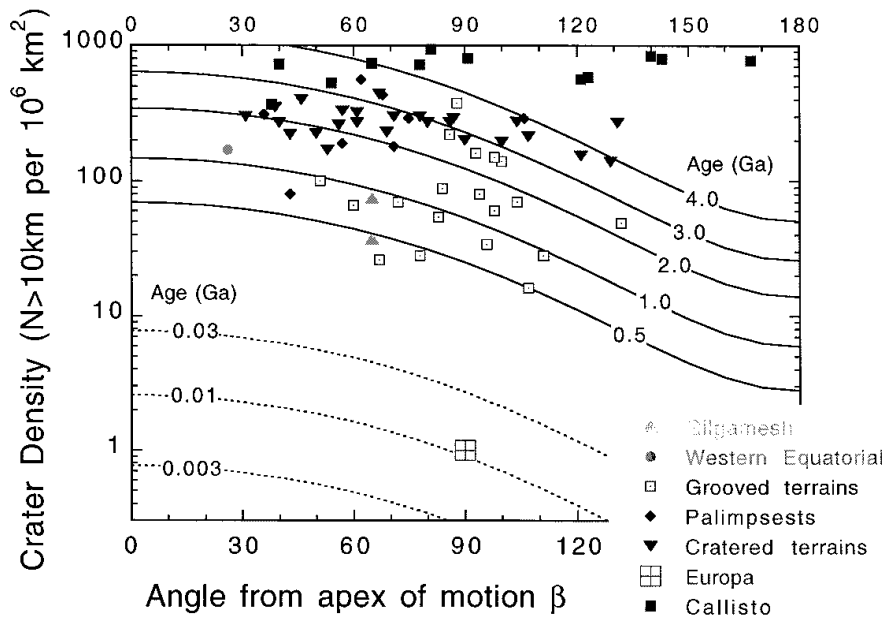

FIG. 7. Crater densities on various types of surfaces on Ganymede and Callisto as a function of the angle $\beta$ from the apex of motion. Data are those tabulated by Shoemaker et al. (1982). All data are expressed in terms of the equivalent number of $10-\mathrm{km}$ craters that would be extrapolated using Shoemaker's $N(>D) \propto D^{-2.2}$ power law. Gilgamesh and the Western Equatorial basin are large young impact basins. The grooved terrains are generally although not always young, while the cratered terrains and palimpsests (ghostly imprints of lost impact basins) are old. The datum for Europa is the nominal equivalent of five $20-\mathrm{km}$ craters scattered over the moon's surface. The curves are the surface ages that correspond to these crater densities at these apex angles-solid curves are ages relevant to Ganymede, the dotted curves are ages relevant to the higher cratering rate at Europa-calculated according to the assumption that the satellites have been in synchronous rotation throughout. The surface ages are those predicted using our nominal cratering rate, with the additional assumption that the Kuiper belt decays as 1/t (Holman and Wisdom 1993). These are thus uncertain by as much as a factor five. Ages for Callisto are not shown, but are consistent with the age of the solar system.

the additional assumption that Ganymede's surface has been locked to Jupiter in synchronous rotation.

Two things are notable about Fig. 7. The first is that many surfaces on Ganymede appear to be considerably younger than the age of the solar system. Many of the grooved terrains appear to be less than a billion years old, some younger than $0.5 \mathrm{Ga}$. From the crater density on its ejecta blanket, Gilgamesh is here assigned a nominal age of $0.7 \mathrm{Ga}$, rather than $3.8 \mathrm{Ga}$. If we extrapolate Shoemaker's $N(>d) \propto d^{-2}$ power law to $50-\mathrm{km}$ comets, Gilgamesh would be a 2-Ga event. The other thing is that apexantapex asymmetries are poorly expressed. What asymmetries there may be are not very pronounced. ${ }^{3}$ That crater densities of the older terrains are all about the same may simply be a ganymedean mainfestation of crater saturation

\footnotetext{
${ }^{3} \mathrm{Or}$, in the case of bright-ray craters on Ganymede, counterintuitive, which Passey and Shoemaker (1982) explain away by pointing out that erosive processes are also faster on the leading hemisphere.
}

or cratering equilibrium. But the younger, clearly unsaturated surfaces also show little evidence of an apex-antapex asymmetry. It is possible that the asymmetry is there in the data, obscured by small numbers (we look forward to seeing crater counts at $\beta \geq 160^{\circ}$ ), but it is also possible that the ice shell has rotated in the past billion years.

Obviously the factor five uncertainty is important to the chronology of Ganymede-it is the difference between young and old. Figure 8 shows cratering ages of various surfaces on Ganymede according to the assumption that Ganymede has rotated nonsynchronously; i.e., we use the global average impact rate at all longitudes. The emphasis of the figure is on the bounds to ages permitted by a factor five uncertainty in current cratering rates. It is possible that Gilgamesh and the other sparsely cratered terrains are as old as $3 \mathrm{Ga}$ (indeed they could be older still if the current cratering rate is unusually high). This is probably not old enough to align Ganymede with the lunar chronology, but it is old. Of course it is also possible, so far as the crater counts are concerned, that large regions of Ganymede are younger than $100 \mathrm{Ga}$-few would tread this path. But overall, it seems to us more plausible that large provinces on Ganymede truly are relatively young. It must be emphasized that, unlike the Moon or even Mars, Ganymede is internally alive. This at least is the inference one draws from its active magnetic field. A surface frozen dead for nearly $4 \mathrm{Ga}$ might well seem the bigger surprise, especially if Ganymede passed through a resonance that invigorated its interior less than 1 Ga ago (Tittemore 1990,

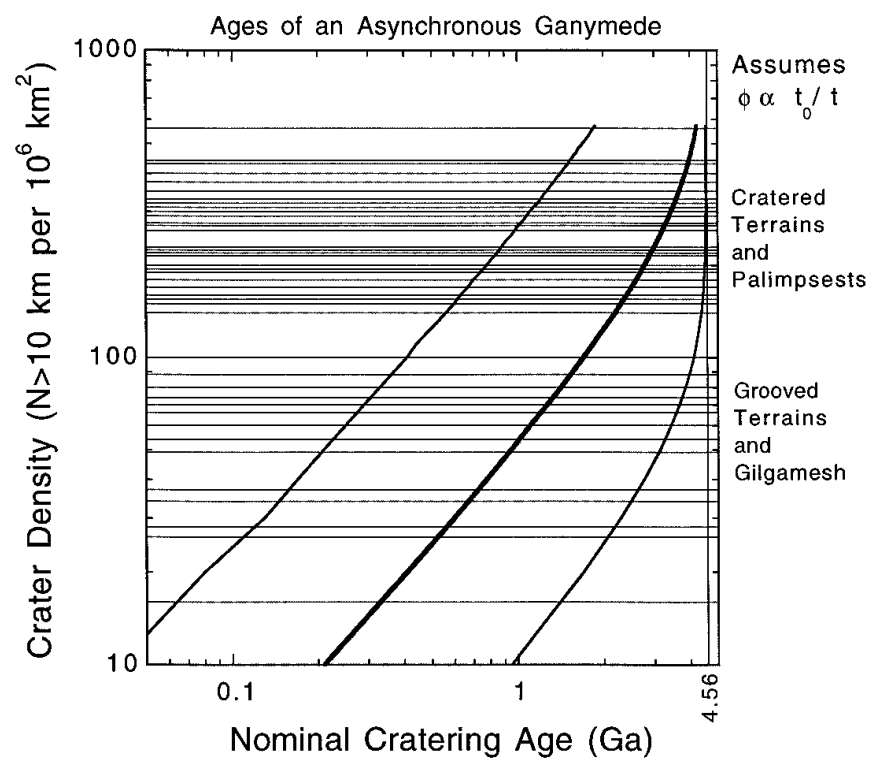

FIG. 8. Crater densities and ages of various surfaces on Ganymede. Ages for the nominal cratering rate, and rates five times higher and lower are shown. This spans the range of formal uncertainty. Here we use only the global average cratering rate to set ages; this is equivalent to Ganymede rotating nonsynchronously. 
Malhotra 1991, Showman and Malhotra 1997, Showman et al. 1997).

Callisto. Callisto poses no obvious problems with respect to apex-antapex asymmetry: Callisto defines what one should mean by a "saturated" or "equilibrium" cratered surface. By any measure its surface is old. It is more heavily cratered than can be explained by a JFC population declining as $t^{-1}$. A higher cratering rate in the early solar system is required. Hence we cannot assign an age to Callisto's surface. It may seem reasonable to regard the higher early cratering rate as being coincident with and sharing a common cause with the late lunar bombardment, but there is no evidence to argue either way. What is strange is that the crater densities on Callisto are significantly higher than they are on the old cratered terrains of Ganymede (see Fig. 7), yet the cratering rate on Callisto is only about half that on Ganymede (Table IV). The difference could be in the physics of crater equilibrium, or it could be that even Ganymede's cratons are relatively young. If we choose the latter, we would also choose nonsynchronous rotation for Ganymede.

\section{SUMMARY}

Impacts at Jupiter are mostly by Jupiter-family comets, with a possibly significant contribution at the smaller sizes by trojan asteroids. Together these populations contribute to terrestrial cratering at no more than the 5-10\% level. Terrestrial cratering is dominated by asteroids with possibly important contributions by long period and Halleytype comets. None of these populations contribute significantly to cratering in the jovian system. In sum, there is no reason to expect correlations between cratering records in the outer solar system and those in the inner solar system. This is not a new observation (cf. SW82), yet it needs to be repeated. The impacting objects are almost wholly different. In particular, there is no reason to apply the lunar chronology to Ganymede.

Io has no craters, and Callisto is old and saturated. Europa and Ganymede are more immediately interesting. Europa has a few 20-km impact craters; from these we conclude that its surface is of the order of $10 \mathrm{Ma}$ old, with an uncertainty of about a factor of five. It is young by all our counts. Ganymede is older, but the younger surfaces have nominal ages of 0.5-1.0 Ga. The Gilgamesh ejecta blanket in particular is assigned a nominal age of $0.7_{-0.55}^{+1.8}$ $\mathrm{Ga}$. Such young ages are consistent with Ganymede being alive, in a geological sense, and are more consistent with Ganymede's currently maintaining an internally generated magnetic field than a more conventionally ancient age would be (Stevenson 1986).

Any lack of apex-antapex asymmetry on Europa could probably be explained away as secondaries or, for the larger craters, as small number statistics, but it is not alto- gether unlikely that the Europan surface moves, either slowly on some glacial timescale, or in response to tidal heating, or catastrophically in the manner of true polar wander. Such phenomena have been predicted (e.g., Greenberg and Weidenschilling 1984) and continue to be seriously discussed (Geissler et al. 1998). Nonsynchronous rotation seems most likely if the ice is really a shell floating on a liquid ocean. It is harder to envision if the water layer is solid ice everywhere. In this context Ganymede again raises deep issues: how, other than by rotation, is Ganymede to avoid a pronounced apex-antapex cratering asymmetry? And if there is no other choice, does this not require that Ganymede too was once, and perhaps not so long ago, home to a liquid ocean?

\section{ACKNOWLEDGMENTS}

We thank the Galileo imaging team for requesting an outside study of this matter. Our thanks to Clark Chapman, Jeff Moore, M. Belton, and Mike Carr. We also thank Erik Asphaug, Diana Blaney, Martin Duncan, Alfred McEwen, Wm. B. McKinnon, Gerhard Neukum, Torrance Johnson, and Paul Schenk, for suggestions, discussions, and invaluable advice. NASA support is courtesy of Exobiology (KZ), Origins (HL), Planetary Geology and Geophysics (LD), and Planetary Atmospheres (KZ). We especially thank Gene Shoemaker. We dedicate this paper to his memory.

\section{REFERENCES}

Anderson, J., E. Lau, W. Sjogren, G. Schubert, and W. Moore 1997. Nature 387, 264-266.

Asphaug, E., and W. Benz 1996. Size, density, and structure of Comet Shoemaker-Levy 9 inferred from the physics of tidal breakup. Icarus 121, 225-248.

Bailey, M. E., and V. V. Emel'yanenko 1996. Dynamical evolution of Halley-type comets. Mon. Not. Roy. Astron. Soc. 278, 1087-1110.

Bailey, M., S. V. M. Clube, G. Hahn, W. M. Napier, and G. B. Valsecchi 1994. Hazards due to giant comets: Climate and short-term catastrophism. In Hazards Due to Comets and Asteroids (T. Gehrels, Ed.), pp. 479-533. Univ. of Arizona Press, Tucson.

Barucci, M. A., M. Lazarin, and M. C. DeSanctis 1996. Comet P/Gehrels 3: Spectroscopic investigations and thermal modelling. Bull. Am. Astron. Soc. 28, 1084.

Blaney, D. L., T. V. Johnson, D. L. Matson, and G. J. Veeder 1995. Volcanic eruptions on Io: Heat flow, resurfacing, and lava composition. Icarus 113, 220-226.

Brandt, J., C. Randall, I. Stewart, M. A'Hearn, Y. Fernandez, and D. Schleicher 1997. The lost tribe of small comets. Bull. Am. Astron. Soc. 29, 1260.

Carr, M., M. J. S. Belton, C. Chapman, M. Davies, P. Geissler, R. Greenberg, A. McEwen, R. Tufts, R. Greeley, R. Sullivan, J. Head, R. Pappalardo, K. Klaasen, T. Johnson, J. Kaufman, D. Senske, J. Moore, G. Neukum, G. Schubert, J. Burns, P. Thomas, and J. Veverka 1998. Evidence for a subsurface ocean of Europa. Nature 391, 363-365.

Carusi, A. L. Kresak, E. Perozzi, and G. B. Valsecchi 1987. High-order librations of Halley-type comets. Astron. Astrophys 187, 899-911.

Cassen, P., S. Peale, and R. Reynolds 1980. On the comparative evolution of Ganymede and Callisto. Icarus 41, 232-239. 
Cellino, A., V. Zappalà, and P. Farinella 1991. The size distribution of main-belt asteroids from IRAS data. Mon. Not. R. Astron. Soc. 253, 561-574.

Chapman, C. R., and W. B. McKinnon 1986. Cratering of planetary satellites. In Satellites (J. A. Burns and M. S. Matthews, Eds.), pp. 507-553. Univ. of Arizona Press, Tucson.

Clarke, A. C. 1982. 2010: Oddysey Two. Ballantine, New York.

Dohnanyi, J. S. 1972. Interplanetary objects in review: Statistics of their masses and dynamics. Icarus 17, 1-48.

Dones, L. 1997. Origin and evolution of the Kuiper Belt. In From Stardust to Planetesimals (Y. J. Pendleton and A. G. G. M. Tielens, Eds.), Astronomical Society of the Pacific Conference Series, Vol. 122, pp. 347-365. Astron. Soc. Pacific, San Francisco.

Donnison, J. R. 1986. The distribution of cometary magnitudes. Astron. Astrophys. 167, 359-363.

Duncan, M. J., and H. F. Levison 1997. A scattered comet disk and the origin of Jupiter family comets. Science 276, 1670-1672.

Duncan, M., T. Quinn, and S. Tremaine 1988. The origin of short-period comets. Astrophys. J. Lett. 328, L69-L73.

Durda, D., and S. F. Dermott 1997. The collisional evolution of the asteroid belt and its contribution to the zodiacal cloud. Icarus 130, 140-164.

Everhart, E. 1967. Intrinsic distributions of cometary perihelia and magnitudes. Astron. J. 72, 1002.

Fernandez J. A., and W.-H. Ip 1991. Statistical and evolutionary aspects of cometary orbits. In Comets in the Post-Halley Era (R. L. Newburn, M. Neugebauer, and J. Rahe, Eds.), pp. 487-535. Kluwer, Dordrecht.

Fernandez, J. A., H. Rickman, and L. Kamél 1992. The population size and distribution of perihelon distances of the Jupiter Family. In Periodic Comets (J. A. Fernandez and H. Rickman, Eds.), pp. 143-157. Univ. de la Republica, Montevideo.

Geissler, P. E., R. Greenberg, G. Hoppa, P. Helfenstein, A. McEwen, R. Pappalardo, R. Tufts, M. Ockert-Bell, R. Sullivan, R. Greeley, M. J. S. Belton, T. Denk, B. Clark, J. Burns, J. Veverka, and the Galileo Imaging Team 1998. Evidence for nonsynchronous rotation of Europa. Nature 391, 368-371.

Gladman, B. J., F. Migliorini, A. Morbidelli, V. Zappalà, P. Michel, A. Cellino, C. Froeschlè, H. Levison, M. Bailey, and M. Duncan 1997. Dynamical lifetimes of objects injected into asteroid belt resonances. Science 277, 197-201.

Greenberg, R., and S. Weidenschilling 1984. How fast do the Galilean satellites spin? Icarus 58, 186-196.

Grieve, R. A. F., and E. M. Shoemaker 1994. The record of past impacts on Earth. In Hazards Due to Comets and Asteroids (T. Gehrels, Ed.), pp. 417-462. Univ. of Arizona Press, Tucson.

Harmon J. K., S. J. Ostro, L. A. M. Benner, K. D. Rosema, and 12 colleagues 1997. Radar detection of the nucleus and coma of Comet Hyakutake (C/1996 B2). Science 278, 1921-1924.

Hoagland, R. C. 1980. The Europa enigma. Star and Sky 2, 16-31.

Holman, M. J., and J. Wisdom 1993. Dynamical stability in the outer Solar System and the delivery of short-period comets. Astron. J. 105, 1987-1999.

Horedt, G. P., and G. Neukum 1984. Cratering rate over the surface of a synchronous satellite. Icarus 60, 710-717.

Hughes, D. W. 1988. Cometary magnitude distribution and the ratio between the numbers of long- and short-period comets. Icarus 73, 149-162.

Jewitt, D. 1991. Cometary photometry. In Comets in the Post-Halley Era (R. L. Newburn, M. Neugebauer, and J. Rahe, Eds.), pp. 19-66. Kluwer, Dordrecht.
Johnson, T. V., and L. A. Soderblom 1982. Volcanic eruptions on Io: Implications for surface evolution and mass loss. In Satellites of Jupiter (D. Morrison, Ed.), pp. 634-646. Univ. of Arizona Press, Tucson.

Kary, D. M., and L. Dones 1996. Capture statistics of short-period comets: Implications for Comet D/Shoemaker-Levy 9. Icarus 121, 207-224.

Kidger, M. 1997. http: //galileo.ivv.nasa.gov/comet/news66.html.

Kresak, L., and M. Kresakova 1989. The absolute magnitudes of periodic comets. I-Catalogue. Bull. Astron. Inst. Czech. 40, 269-284.

Kresak, L., and E. M. Pittich 1978. The intrinsic number density of active long-period comets in the inner Solar System. Astron. Inst. Czech. Bull. 29, 299-309.

Kronk, G. W. 1984. Comets, a Descriptive Catalog. Enslow, Hillside, NJ.

Lamy, P. L., I. Toth, L. Jorda, H. A. Weaver, and M. A'Hearn 1998. The nucleus and inner coma of Comet 46P/Wirtanen. Astron. Astrophys. 335, L25-L29.

Levison, H. F. 1996. Comet taxonomy. In Completing the Inventory of the Solar System (T. W. Rettig and J. M. Hahn, Eds.), pp. 173-191. Astron. Soc. Pac. Conf. Proc., vol. 107.

Levison, H., and M. Duncan 1994. The long-term dynamical behavior of short-period comets. Icarus 108, 18-36.

Levison, H., and M. Duncan 1997. From the Kuiper Belt to Jupiter-family comets: The spatial distribution of ecliptic comets. Icarus 127, 13-32.

Levison, H., E. M. Shoemaker, and C. S. Shoemaker 1997. The dispersal of the Trojan asteroid swarm. Nature 385, 42-44.

Malhotra, R. 1991. Tidal origin of the Laplace resonance and the resurfacing of Ganymede. Icarus 94, 399-412.

Marzari, F., P. Farinella, and V. Vanzani 1995. Are Trojan collisional families a source for short-period comets? Astron. Astrophys. 299, 267.

McEwen, A. S., J. M. Moore, and E. M. Shoemaker 1997. The Phanerozoic impact cratering rate: Evidence from the farside of the Moon. J. Geophys. Res. 102, 9231-9242.

McKinnon, W. B., C. R. Chapman, and K. R. Housen 1991. Cratering of the uranian satellites. In Uranus (J. T. Bergstrahl, E. D. Miner, and M. S. Matthews, Eds.), pp. 629-692. Univ. of Arizona Press, Tucson.

Melosh, H. J. 1989. Impact Cratering: A Geological Process. Oxford Univ. Press, New York.

Nakamura, T., and M. Yoshikawa 1995. Close encounters and collisions of short-period comets with Jupiter and its satellites. Icarus 116, 113-130.

Neukum, G., R. Wagner, U. Wolf, J. Head, R. Pappalardo, C. Chapman, W. Merline, and M. Belton 1997. Bombardment history of the Galilean satellites and derived ages. Bull. Am. Astron. Soc. 29, 984.

Olsson-Steel, D. 1987. Collisions in the Solar System-IV. Cometary impacts on the planets. Mon. Not. R. Astron. Soc. 227, 501-524.

Pappalardo, R., J. Head, R. Greeley, R. Sullivan, C. Pilcher, G. Schubert, W. B. Moore, M. Carr, J. Moore, M. J. S. Belton, and D. L. Goldsby 1998. Geological evidence for solid-state convection in Europa's ice shell. Nature 391, 365-368.

Passey, Q. R., and E. M. Shoemaker 1982. Craters and basins on Ganymede and Callisto. In Satellites of Jupiter (D. Morrison, Ed.), pp. 379-434. Univ. of Arizona Press, Tucson.

Rabe, E. 1972. Orbital characteristics of comets passing through the 1:1 commensurability with Jupiter. In The Motion, Evolution of Orbits, and Origin of Comets, IAU Symposium 45 (G. A. Chebotarev, E. I. Kazimirchak-Polonskaya, and B. G. Marsden, Eds.), pp. 156-166. Reidel, Dordrecht.

Rahe, J., V. Vanysek, and P. R. Weissman 1994 Properties of cometary nuclei. In Hazards Due to Comets and Asteroids (T. Gehrels, Ed.), pp. 597-634. Univ. Arizona Press, Tucson.

Reynolds, R. T., S. Squyres, D. Colburn, and C. P. McKay 1983. On the habitability of Europa. Icarus 56, 246-254. 
Roemer, E., M. Thomas, and R. E. Lloyd 1966. Observations of comets, minor planets, and Jupiter VIII. Astron J. 71, 591.

Safronov, V. S. 1972. Evolution of the Protoplanetary Cloud and Formation of the Earth and the Planets, NASA Tech. Transl. F-677.

Schmidt, R. M., and K. R. Housen 1987. Some recent advances in the scaling of impact and explosive cratering. Intl. J. Impact Eng. 5, 543-560.

Scotti, J. 1998. Periodic comet extreme observations. http://pirl.lpl. arizona.edu/ jscotti/comets.dir/comext.ps

Sekanina, Z., and D. K. Yeomans 1985. Orbital motion, nuclear precession, and splitting of periodic Comet Brooks 2. Astron. J. 90, 2335-2352.

Shoemaker, E. M. 1996. The age of Europa's surface. In Europa Ocean Conference (D. Matson and D. Nash, Eds.), pp. 65-66. Nov. 12-14, 1996. San Juan Capistrano, California. http://www.sji.org/conf/Europa.html

Shoemaker, E. M., and R. A. Wolfe 1982. Cratering timescales for the Galilean satellites. In Satellites of Jupiter (D. Morrison, Ed.), pp. 277339. Univ. of Arizona Press, Tucson.

Shoemaker, E. M., B. K. Lucchitta, D. E. Wilhelms, J. B. Plescia, and S. W. Squyres 1982. The geology of Ganymede. In Satellites of Jupiter (D. Morrison, Ed.), pp. 435-520. Univ. of Arizona Press, Tucson.

Shoemaker, E. M., P. R. Weissman and C. S. Shoemaker 1994. The flux of periodic comets near Earth. In Hazards Due to Comets and Asteroids (T. Gehrels, Ed.), pp. 313-335. Univ. of Arizona Press, Tucson.

Shoemaker, E. M., R. F. Wolfe, and C. S. Shoemaker 1990. Asteroid and comet flux in the neighborhood of Earth. In Global Catastrophes in Earth History (V. L. Sharpton and P. D. Ward, Eds.), pp. 155-170. Geological Soc. of America Special Pap. 247, Boulder.

Showman, A., and R. Malhotra 1997. Tidal evolution into the LaPlace resonance and the resurfacing of Ganymede. Icarus 127, 93-111.

Showman, A., D. J. Stevenson, and R. Malhotra 1997. Coupled orbital and thermal evolution of Ganymede. Icarus 129, 367-383.

Steel, D. I. 1993. Collisions in the Solar System: V. Terrestrial impact probabilities for parabolic comets. Mon. Not. R. Astron. Soc. 264, 813.

Stern, S. A., and J. Colwell 1997. Accretion in the Edgeworth-Kuiper belt: Forming 100-1000 km radius bodies at $30 \mathrm{AU}$ and beyond. Astron. J. 114, 841-849.

Stevenson, D. J. 1996. When Galileo met Ganymede. Nature 384, $511-512$.

Sullivan, R., R. Greeley, K. Homan, J. Klemaszewski, M. J. S. Belton, M. Carr, C. Chapman, R. Tufts, J. Head, R. Pappalardo, J. Moore, P. Thomas, and the Galileo Imaging Team 1998. Episodic plate separation and fracture infill on the surface of Europa. Nature 391, 371-373.

Sykes, M. V., and R. G. Walker 1992. Cometary dust trails. Icarus 95, 180-210.

Tanaka, H., S. Inaba, and K. Nakazawa 1996. Steady-state size distribution for the self-similar cascade. Icarus 123, 450-455.

Tittemore, W. C. 1990. Chaotic motion of Europa and Ganymede and the Ganymede-Callisto dichotomy. Science 250, 263-267.

Weaver, H. A., P. D. Feldman, M. F. A'Hearn, C. Arpigny, and 6 colleagues 1997. The activity and size of the nucleus of Comet Hale-Bopp (C/1995 O1). Science 275, 1900-1904.

Weissman, P. R. 1990. The cometary impactor flux at the Earth. In Global Catastrophes in Earth History (V. L. Sharpton and P. D. Ward, Eds.), pp. 171-180. Geological Soc. of America Special Pap. 247, Boulder.

Weissman, P. R. 1991. Dynamical history of the Oort cloud. In Comets in the Post-Halley Era (R. L. Newburn, M. Neugebauer, and J. Rahe, Eds.), pp. 463-486. Kluwer, Dordrecht.

Weissman, P. R. and H. Levison 1997. Origin and evolution of the unusual object 1996PW: An asteroid from the Oort cloud? Astrophys. J. Lett. 488, 133-136.

Wetherill, G. 1994. The provenence of the terrestrial planets. Geochim. Cosmochim. Acta 58, 4513-4524.

Wiegert, P., and S. Tremaine 1998. The evolution of long-period comets. Icarus, in press.

Williams, D. R., and G. W. Wetherill 1994. Size distribution of collisionally evolved asteroidal populations: Analytical solution for self-similar collision cascades. Icarus 107, 117-128.

Zahnle, K. 1998. Origins of atmospheres. In Origins (C. Woodward, J. M. Shull, and H. Thronson, Eds.), Astron. Soc. Pacific Conf. Series, Vol. 148. pp. 364-391. San Francisco. 